\title{
Influenza virus infection causes global RNAPII termination defects
}

\author{
Nan Zhao 1,2,22, Vittorio Sebastiano 3,4,22, Natasha Moshkina',2,22, Nacho Mena ${ }^{1,2}$, Judd Hultquist ${ }^{5}$, \\ David Jimenez-Morales ${ }^{6}$, Yixuan Ma ${ }^{1,2}$, Alex Rialdi ${ }^{1,2}$, Randy Albrecht ${ }^{10^{1,2}}$, Romain Fenouil7, \\ Maria Teresa Sánchez-Aparicio, ${ }^{1,2}$, Juan Ayllon ${ }^{1,2}$, Sweta Ravisankar ${ }^{3,4}$, Bahareh Haddad ${ }^{3,4}$, \\ Jessica Sook Yuin $\mathrm{Ho}^{8}$, Diana Low ${ }^{8}$, Jian Jin ${ }^{9}{ }^{9}$, Vyacheslav Yurchenko ${ }^{10}$, Rab K. Prinjha ${ }^{11}$, \\ Alexander Tarakhovsky ${ }^{12}$, Massimo Squatrito ${ }^{13}$, Dalila Pinto ${ }^{14}{ }^{14}$, Kimaada Allette ${ }^{15}$, Minji Byun ${ }^{16}$, \\ Melissa Laird Smith ${ }^{15}$, Robert Sebra ${ }^{15}$, Ernesto Guccione ${ }^{8}$, Terrence Tumpey ${ }^{17}$, Nevan Krogan ${ }^{6}$, \\ Benjamin Greenbaum ${ }^{18,19,20}$, Harm van Bakel ${ }^{7}$, Adolfo García-Sastre ${ }^{1,2,21,23}$ and Ivan Marazzi ${ }^{10,2,23 \star}$
}

\begin{abstract}
Viral infection perturbs host cells and can be used to uncover regulatory mechanisms controlling cellular responses and susceptibility to infections. Using cell biological, biochemical, and genetic tools, we reveal that influenza A virus (IAV) infection induces global transcriptional defects at the $3^{\prime}$ ends of active host genes and RNA polymerase II (RNAPII) run-through into extragenic regions. Deregulated RNAPII leads to expression of aberrant RNAs (3' extensions and host-gene fusions) that ultimately cause global transcriptional downregulation of physiological transcripts, an effect influencing antiviral response and virulence. This phenomenon occurs with multiple strains of IAV, is dependent on influenza NS1 protein, and can be modulated by SUMOylation of an intrinsically disordered region (IDR) of NS1 expressed by the 1918 pandemic IAV strain. Our data identify a strategy used by IAV to suppress host gene expression and indicate that polymorphisms in IDRs of viral proteins can affect the outcome of an infection.
\end{abstract}

\begin{abstract}
$\mathrm{AV}$ is a negative-sense single-stranded RNA virus. The IAV genome is organized into eight segments, short negative-sense noncoding 'mini RNA chromosomes' that are packaged inside virions after the virus completes a life cycle in infected cells. IAV, like all viruses, is an obligate parasite, because it requires cellular functions to multiply and disseminate. However, unlike most other RNA viruses, it replicates in the nucleus, a feature that places evolutionary pressure on a number of viral proteins to coopt or interfere with host chromatin-based regulatory processes in infected cells ${ }^{1,2}$.

All IAV strains encode a nonstructural protein called NS1, whose function is to antagonize host antiviral responses ${ }^{3}$. NS1mediated host antagonism occurs through multiple mechanisms, including inhibition of sensing of the virus ${ }^{4}$ and suppression of host
\end{abstract}

functions that are detrimental to the virus, such as host translation and inflammatory-gene expression ${ }^{1,6}$. NS1 protein sequences differ among strains. Only a few domains of NS1, such as the RNAbinding domain ${ }^{7}$ and the $\mathrm{C}$-terminal domain, have been identified as pathogenic determinants in multiple strains ${ }^{8}$. Interestingly, the $\mathrm{C}$ terminus is among the most divergent sequences within NS1, and it is unstructured ${ }^{1,9,10}$. These features are reminiscent of IDRs, or unstructured domains often referred to as short linear modules (SLIMs), peptide motifs, or linear domains ${ }^{11}$. Pathogens often use IDRs to promote novel interactions to adapt to new hosts and to enhance transmission and virulence ${ }^{12}$.

In this work, we used influenza 1918 virus $^{13,14}$, a pandemic IAV that has caused the worst pandemic known to date ${ }^{15}$, as a case study

'Department of Microbiology, Icahn School of Medicine at Mount Sinai, New York, NY, USA. ${ }^{2}$ Global Health and Emerging Pathogens Institute, Icahn School of Medicine at Mount Sinai, New York, NY, USA. ${ }^{3}$ Department of Obstetrics and Gynecology, Stanford University, Stanford, CA, USA. ${ }^{4}$ Institute for Stem Cell Biology \& Regenerative Medicine, Stanford University, Stanford, CA, USA. ${ }^{5}$ Department of Medicine (Infectious Diseases), Northwestern University Feinberg School of Medicine, Chicago, IL, USA. ${ }^{6}$ Department of Cellular and Molecular Pharmacology, University of California, San Francisco, San Francisco, CA, USA. ${ }^{7}$ Icahn Institute for Genomics and Multiscale Biology, Icahn School of Medicine at Mount Sinai, New York, NY, USA. ${ }^{8}$ Institute of Molecular and Cell Biology (IMCB), Agency for Science, Technology and Research (A*STAR), Singapore, Singapore. ${ }^{9}$ Mount Sinai Center for Therapeutics Discovery, Departments of Pharmacological Sciences and Oncological Sciences, Tisch Cancer Institute, Icahn School of Medicine at Mount Sinai, New York, NY, USA. ${ }^{10}$ Life Science Research Centre, Faculty of Science, University of Ostrava, Ostrava, Czech Republic. ${ }^{11}$ Epinova Epigenetics Discovery Performance Unit, Immuno-Inflammation Therapy Area, GlaxoSmithKline, Medicines Research Centre, Stevenage, UK. ${ }^{12}$ Laboratory of Immune Cell Epigenetics and Signaling, The Rockefeller University, New York, NY, USA. ${ }^{13}$ Cancer Cell Biology Programme, Centro Nacional de Investigaciones Oncológicas, CNIO, Madrid, Spain. ${ }^{14}$ Department of Psychiatry, Department of Genetics and Genomic Sciences, Icahn School of Medicine at Mount Sinai, New York, NY, USA. ${ }^{15}$ Department of Genomics and Multiscale Biology, Icahn School of Medicine at Mount Sinai, New York, NY, USA. ${ }^{16}$ Department of Medicine, Clinical Immunology, Icahn School of Medicine at Mount Sinai, New York, NY, USA. ${ }^{17}$ Influenza Division, National Center for Immunization and Respiratory Diseases, Centers for Disease Control and Prevention, Atlanta, GA, USA. ${ }^{18}$ Tisch Cancer Institute, Icahn School of Medicine at Mount Sinai, New York, NY, USA. ${ }^{19}$ Division of Hematology and Oncology, Department of Medicine, Icahn School of Medicine at Mount Sinai, New York, NY, USA. ${ }^{20}$ Department of Pathology, Icahn School of Medicine at Mount Sinai, New York, NY, USA. ${ }^{21}$ Division of Infectious Diseases, Department of Medicine, Icahn School of Medicine at Mount Sinai, New York, NY, USA. ${ }^{22}$ These authors contributed equally to this work: Nan Zhao, Vittorio Sebastiano, Natasha Moshkina. ${ }^{23}$ These authors jointly directed this work: Adolfo García-Sastre, Ivan Marazzi. *e-mail: ivan.marazzi@mssm.edu 
to identify novel features of IAV-host interaction and adaptation mediated by IDRs. We found that 1918 IAV encodes an NS1 that contains a unique C-terminal domain composed of a small ubiquitin-like modifier (SUMO) site embedded in a PDZ-binding domain (PDZBD) consensus sequence. This extended SUMO consensus or SUMO inside PDZBD (SUP) domain predicts site-specific SUMOylation of 1918 NS1 during infection. Using viruses bearing 1918 NS1, we discovered that IAV elicits global deregulation of RNAPII transcription termination by impairing 3 '-end cleavage and termination. This effect is augmented by NS1 SUMOylation, which increases the partitioning of NS1 in nuclear granules containing 3 '-end-cleavage factors. Termination defects lead to RNAPII traveling through intergenic regions and causing formation of aberrant mRNAs, thus ultimately resulting in global transcriptional downregulation. Analysis of host transcriptional responses to nonpandemic IAV indicated that IAV-induced 3 '-end-termination defects are a general feature of IAV infection that is dependent on NS1 expression and is modulated by post-translational modification of the unstructured region of NS1. Our data support the idea that the study of viral polymorphic proteins can reveal important molecular events occurring during infection.

\section{Results}

A unique domain present in the NS1 of 1918 influenza virus. Along with its structural proteins, IAV encodes the nonstructural protein NS1, which is an antagonist of cellular antiviral responses $^{16,17}$. We surveyed highly pathogenic IAV strains for the presence of unique protein domains and found a unique sequence present in the C-terminal domain of NS1 from the Brevig/mission strain (Fig. 1a and Supplementary Fig. 1a). This strain is responsible for the pandemic influenza outbreak that occurred in 1918 $\left(\right.$ ref. $\left.{ }^{14}\right)$. Interestingly, different strains of IAV bear divergent NS1 C termini (NS1 tail) (Fig. 1b and Supplementary Fig. 1a,b), and NS1 tails have been linked to host tropism and virulence ${ }^{8,18}$. The C terminus of NS1 encoded by the 1918 pandemic strain (hereafter referred to as NS1) ends with the sequence 226-IKSEV-230 (Fig. 1a). This sequence is a SUMOylation consensus site ( $\psi \mathrm{KxE})$ embedded in a PDZBD (Fig. 1a). We examined whether this domain might be a functional acceptor site for SUMO conjugation. K227 was found to be modified by SUMO, whereas the only other canonical SUMO consensus site in position 68-71 was not (Fig. 1c). To verify whether the domain might confer SUMOylation to a noncognate NS1, we generated chimeric NS1 proteins bearing residues 1-225 of H3N2 NS1 (A/New York/739/1994) and residues $226-230$ of 1918 or 1918 -like sequences from avian isolates. This tail swapping conferred SUMOylation in trans (Fig. 1d). We then assessed whether NS1 might be modified during infection. To do so, we generated, through reverse genetics ${ }^{19}$, a chimeric virus with the first seven segments of the A/Puerto Rico/8/1934 H1N1 strain (PR8) and the eighth segment of the 1918 strain (reassortant 7+1, hereafter referred to as NS1 virus). We used this virus to infect A549 human epithelial cells. Immunoprecipitation (IP) of NS1 followed by western blotting (WB) with anti-NS1 and anti-SUMO2/3 revealed that NS1 was SUMOylated during infection (Fig. 1e). This experiment also suggested that NS1 SUMOylation is controlled by SUMO-deconjugating enzymes, because the NS1 modification was lost after omission of the SUMOprotease inhibitor $\mathrm{N}$-ethylmaleimide (NEM) from the cell extracts. Overall, our results indicated that the C terminus of NS1 from the 1918 strain was modified by SUMO within the consensus sequence embedded in a PDZBD. The modified NS1 domain is short and present in an unstructured region of the protein ${ }^{1,9}$, and it can be transferred to other substrates to confer SUMO conjugation. All these features are characteristics of SLIMs and peptide domains ${ }^{20}$. We therefore refer to this domain as the SUP SLIM or SUP peptide domain.
Rescue and analysis of 1918 NS1-SUMO fusion virus. The observation that the PDZBD was modified by a bulky modification (SUMO, with molecular weight $>10 \mathrm{KDa}$ ) suggests that SUMO conjugation might alter the PDZBD-PDZ protein interaction. Leveraging the PDZBD's location at the C terminus of NS1, we engineered an NS1 covalently fused with SUMO (NS1-SUMO) (Fig. 2a). This strategy has been used to identify ubiquitin-dependent mechanisms in histone biology ${ }^{21}$. We also mutated K227 to prevent SUMO conjugation (protein denoted NS1-KR) (Fig. 2a). These mutations did not change the NS1 nuclear localization (Supplementary Fig. 2a). Using biolayer interferometry (BLI), we then determined the $K_{\mathrm{d}}$ of the interaction between a prototypical PDZ-domain-containing protein, PSD95, and the three NS1 variants (wild type (WT), R227, and SUMO). Our results showed that the $K_{\mathrm{d}}$ values of the three NS1 proteins with PSD95 were on the same order of magnitude (1.60 for NS1, 2.30 for NS1-KR, and $0.63 \mu \mathrm{M}$ for NS1-SUMO; Fig. 2a). These results were corroborated by pulldown assays (Supplementary Fig. 2b) and by modeling of NS1 binding with three PDZ proteins (PSD95, GIPC2, and DVL1; Supplementary Fig. 2c-e and Supplementary Table 1). Overall, our results indicated that NS1 retains PDZ interactions independently of its conjugation with SUMO and suggested that SUMOylation has functions other than altering PDZ-PDZ interactions.

To probe the intrinsic role of SUMOylation of the 1918 SUP domain during infection, we engineered two viruses expressing NS1 and NS1-SUMO (hereafter referred to as NS1-SUMO virus) (Fig. 2b). We predicted that generation of the NS1-SUMO virus would be safe because fusion of SUMO to NS1 would result in decreased virulence, similarly to other NS1 fusion viruses ${ }^{22}$. For the opposite reason, the KR-mutant virus was not rescued. Indeed, as demonstrated by hemagglutination assays, the fusion of SUMO to NS1 severely impaired plaque formation (Fig. 2c). Plaque sequencing indicated that the delayed growth of the NS1-SUMO virus at low multiplicity of infection (MOI) was associated with a reversion to its WT virotype (Supplementary Fig. 2f). The decreased virulence of NS1-SUMO virus may have been driven by the formation of many defective particles, impaired assembly, inefficient cellto-cell spreading, or indirect effects caused by SUMOylation affecting NS1 levels (Supplementary Fig. 3a).

Despite having lower pathogenicity, the NS1-SUMO virus still retained the ability to transiently infect at high MOIs. This feature allowed us to monitor early events independent of viral budding, egress, and reinfection of nearby cells. We therefore compared NS1 and NS1-SUMO viruses in experiments conducted at high MOI. A549 cells were infected with NS1 or NS1-SUMO viruses at an MOI of 3, and total RNA was extracted at 6 and $12 \mathrm{~h}$ postinfection (hpi). RNA-seq analysis showed that NS1 and NS1-SUMO RNA levels were comparable (Supplementary Fig. 3b). Both viruses caused altered host gene expression of approximately 1,000 shared genes (Fig. 2d,e), including upregulation of genes that are part of the host defense to infection, in agreement with the high induction of cellular-response genes after 1918 infection $^{23}$. Notably, infection caused the downregulation of most transcriptionally active genes, which accounted for approximately two-thirds of genes whose expression was altered during infection (Fig. 2d). Compared with the NS1 virus, the NS1-SUMO virus displayed a higher magnitude of gene expression changes in both induced and suppressed genes at both 6 and 12 hpi (Fig. 2d). Importantly, both viruses upregulated and downregulated the same set of genes (Fig. 2e). Overall, these results suggest that NS1 affects the magnitude of induction and suppression of host genes.

SUMOylation controls NS1 oligomeric assembly and partitioning in RNA granules. NS1 binds $\mathrm{RNA}^{24}$ and forms polymers in vitro $^{25,26}$. Therefore, we investigated a potential role of NS1 in interaction with RNA granules. These compartments are nucleated by 
a

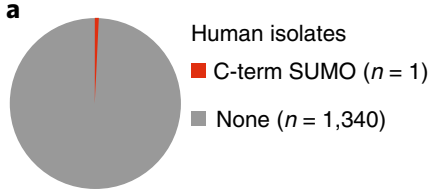

NS1 (1918)

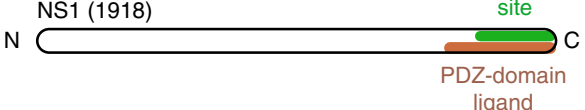

C

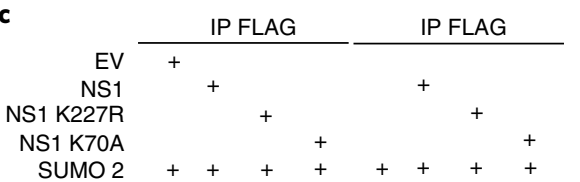

SUMO 2

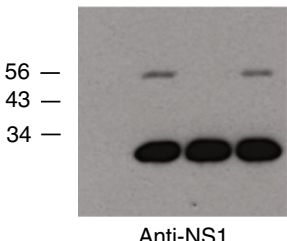

Anti-NS1

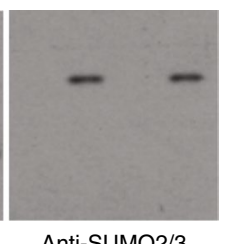

e

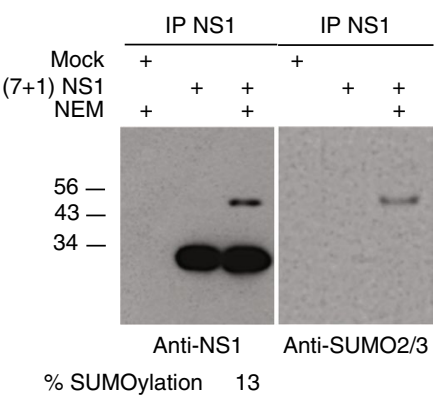

b

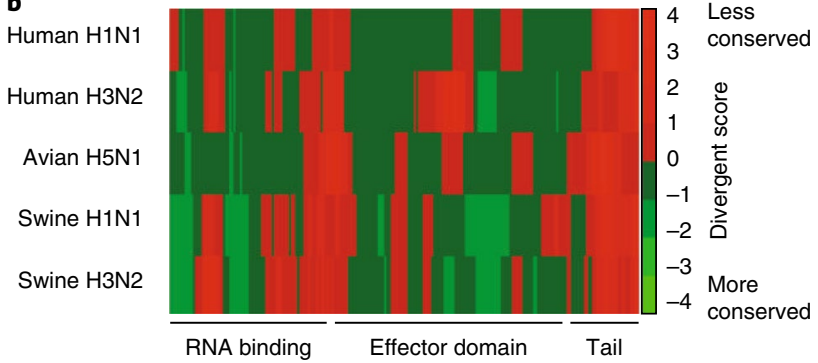

d

\begin{tabular}{|c|c|c|c|c|}
\hline NS1 & N-term & C-term & C-term & $\begin{array}{c}\text { SUMO } \\
\text { site }\end{array}$ \\
\hline 1 & No transfection & - & - & - \\
\hline 2 & $\begin{array}{c}\text { A/Brevig } \\
\text { Mission/1/1918 (H1N1) }\end{array}$ & $\begin{array}{c}\text { A/Brevig Mission/1/1918 } \\
\text { (H1N1)_wild type }\end{array}$ & IKSEV & Yes \\
\hline 3 & $\begin{array}{c}\mathrm{A} / \text { New York/739/1994 } \\
\text { (H3N2) }\end{array}$ & $\begin{array}{c}\text { A/Brevig Mission/1/1918 } \\
\text { (H1N1)_wild type }\end{array}$ & IKSEV & Yes \\
\hline 4 & $\begin{array}{c}\mathrm{A} / \text { New York/739/1994 } \\
\text { (H3N2) }\end{array}$ & $\begin{array}{c}\text { A/Quail/Hong } \\
\text { Kong/AF157/92(H9N2)_wild type }\end{array}$ & IKPEV & Yes \\
\hline 5 & $\begin{array}{c}\mathrm{A} / \text { New York/739/1994 } \\
\text { (H3N2) }\end{array}$ & $\begin{array}{c}\text { A/chicken/Indonesia/CDC25/2005 } \\
\text { (H5N1)_wild type }\end{array}$ & TKSEV & No \\
\hline 6 & $\begin{array}{c}\text { A/Brevig } \\
\text { Mission/1/1918 (H1N1) }\end{array}$ & $\begin{array}{c}\text { A/Brevig Mission/1/1918 } \\
\text { (H1N1)_K227R mutation }\end{array}$ & IRSEV & No \\
\hline
\end{tabular}
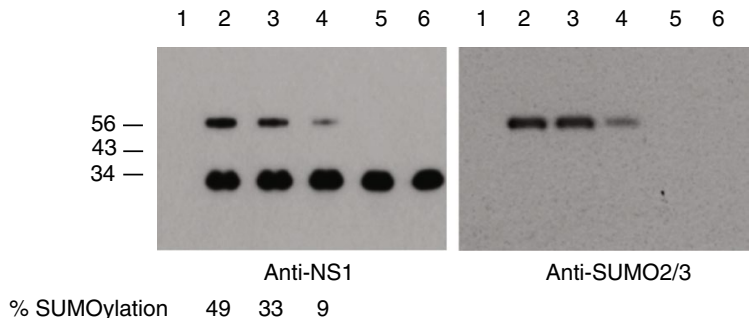

Anti-SUMO2/3

$\begin{array}{llll}\% \text { SUMOylation } \quad 49 & 33 & 9\end{array}$

Fig. 1 | NS1 from 1918 pandemic influenza virus is SUMOylated in its unique C-terminal domain. a, Top, Venn diagram of influenza virus NS1 proteins from human isolates and the presence of a SUMO site in the C-terminal (term) domain (tail). Bottom, amino acid sequence of the unique NS1 tail from A/Brevig Mission/1/1918 (H1N1) bearing a SUMOylation site and PDZ-domain ligand site. $\mathbf{b}$, Conservation plot of amino acid sequences of NS1 among different viral isolates and hosts. The color coding represents the difference between the conservation score and the average value of the score across the protein. Protein domains of NS1 are shown at bottom. c, Ectopic expression of SUMO2 and the indicated NS1 proteins (WT NS1 and the SUMO-consensus mutants at positions K70 and K227) in A549 cells. IP with anti-FLAG and WB analysis with anti-NS1 and anti-SUMO2/3 are shown. d, IP with anti-NS1 and WB with anti-NS1 and anti-SUMO2/3 in A549 cells transfected with the indicated NS1 proteins. The tail swapping of H3N2 NS1 was performed with the sequences from the indicated viruses, all of human origin, except Hong Kong 1992 and Indonesia 2005, which are avian viruses. e, IP and anti-NS1 WB of whole cellular extract from A549 cells infected with the reassortant NS1 virus bearing segment 8 encoding 1918 NS1 (additional data in Fig. 2b). NEM, SUMO peptidase inhibitor. Uncropped blot images are shown in Supplementary Dataset 1.

RNA-binding and aggregation-prone proteins ${ }^{27,28}$ that are regulated by post-translational modifications, such as phosphorylation $^{29}$, SUMOylation ${ }^{30}$, and possibly many more. SUMO enhances the valency and strength of interaction between scaffold and client proteins of RNA granules ${ }^{30}$. We therefore performed semidenaturing detergent agarose gel electrophoresis (SDD-AGE), which allows for the resolution of high-molecular-weight complexes formed as a result of protein multimerization, a feature that can influence partitioning into RNA granules. Results from this assay indicated that NS1 forms high-molecular-weight complexes during infection, and this feature is enhanced by SUMO fusion (Fig. 3a). We then took advantage of the synthetic compound biotinylated isoxazole (B-isox), which has been shown to selectively precipitate RNAgranule components ${ }^{31}$. We synthesized B-isox (Supplementary Fig. $4 a, b)$ and performed selective precipitation from total cell extracts derived from control and infected cells. We thereby confirmed previous findings indicating that $\mathrm{B}$-isox treatment induces the selective precipitation of RNA-granule proteins in a dose-dependent manner ${ }^{31}$ (Supplementary Fig. 4c,d and Supplementary Table 2). We then performed quantitative mass spectrometry analysis, which indicated that infection affected RNA-granule composition (Supplementary Table 3). SUMOylation of NS1 increased partitioning of NS1 in RNA granules (Fig. 3b) and interaction with proteins involved in mRNA processing (Fig. 3c). These findings were further supported by validation experiments, which showed NS1-SUMO-dependent enrichment with the cleavage and polyadenylation specificity factor (CPSF) and the absence of enrichment of other proteins known to be present in RNA granules (FUS and EWS) (Fig. 3c and Supplementary Table 3). Because NS1 proteins from other viral strains are known to antagonize antiviral host gene expression ${ }^{1,3,16,17}$, and recent findings indicate that phase-separated RNA granules play a key role in controlling RNAPII ${ }^{29,32-35}$, we investigated the relationship between NS1 expression and gene suppression by determining RNAPII activity and mRNA levels after infection with 1918 viruses and nonpandemic strains proficient and deficient in NS1. 
a

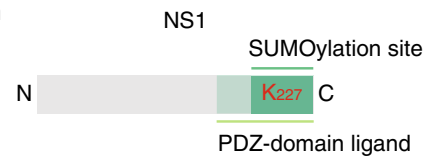

Association

Dissociation

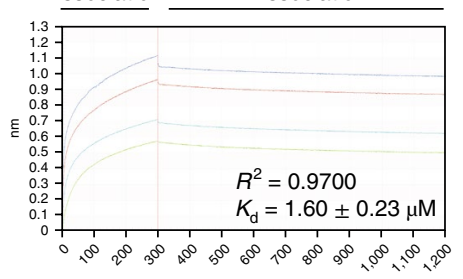

Time (s)

b

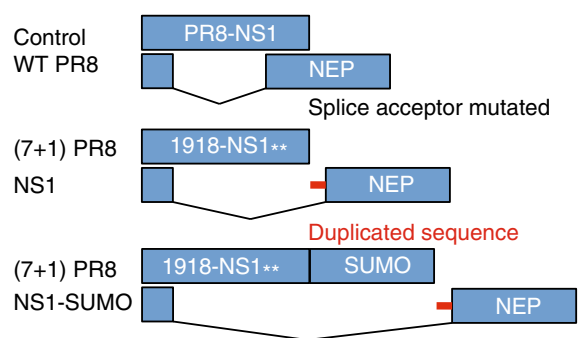

d

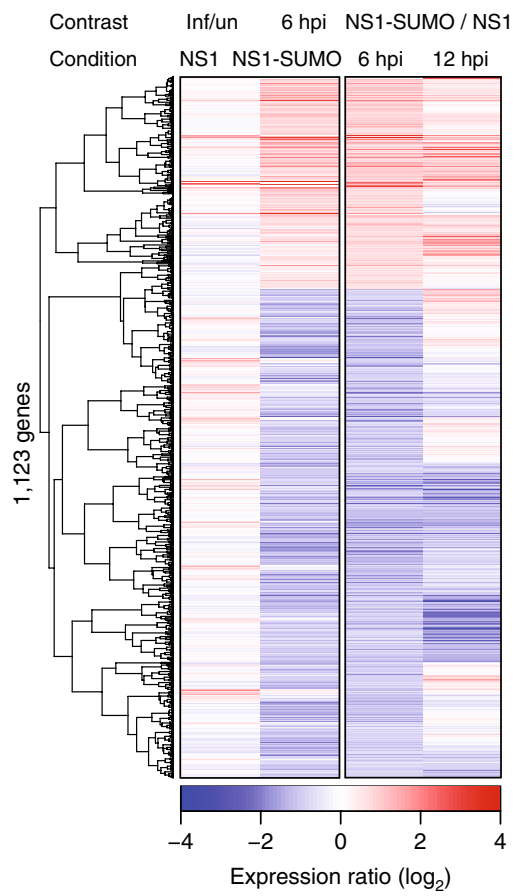

NS1-KR

SUMOylation site

N

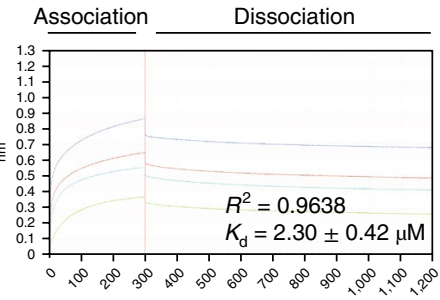

Time (s)

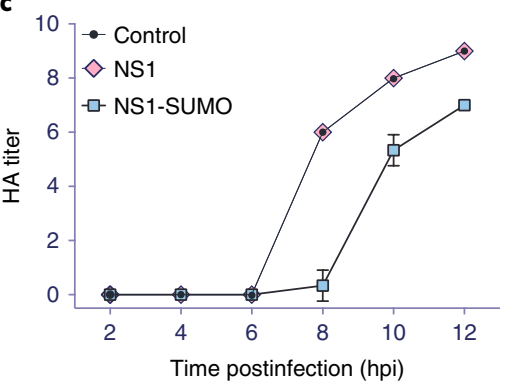

NS1-SUMO

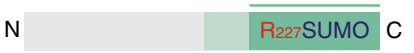

PDZ-domain ligand

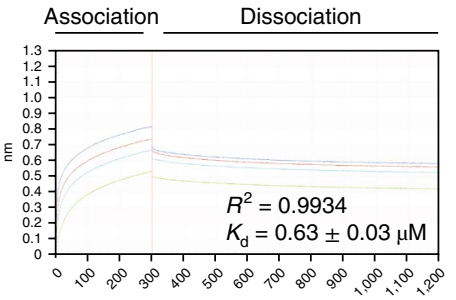

Time (s)

Plaque-size phenotype

Control

WT PR8

(7+1) PR8

NS1

(7+1) PR8 NS1-SUMO

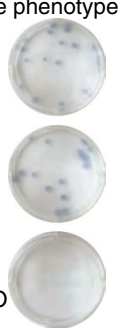

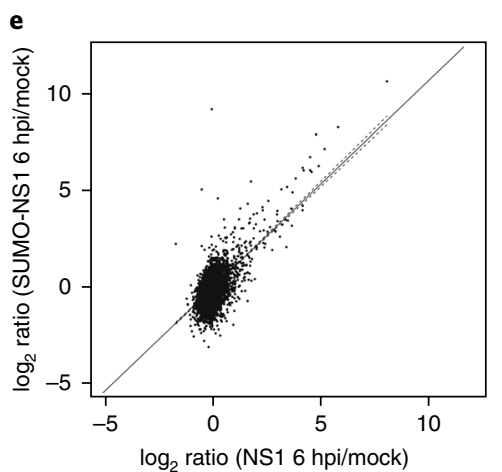

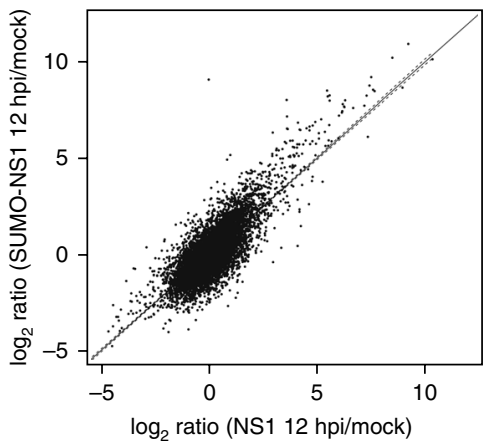

Fig. 2 | 1918 NS1 WT and mutant viruses. a, Top, schematic representations of the recombinant NS1, NS1-KR, and NS1-SUMO proteins used for BLI. Bottom, spectra showing BLI measurements of the interactions between recombinant PSD95 and WT NS1, or NS1 mutants (NS1-KR and NS1-SUMO). b, Schematic representation of WT PR8, the reassortant virus (7+1) PR8 NS1 bearing seven segments of WT PR8 and segment 8 of the 1918 influenza virus, and the reassortant virus (7+1) PR8 NS1-SUMO bearing segment 8 of the 1918 influenza virus encoding a fusion protein between NS1 and SUMO2. c, Growth curves of the indicated viruses after infection of MDCK cells at MOI $=1(n=6)$. Data are plotted as mean \pm s.e.m. of $n=3$ infection experiments. Plaque size from one representative experiment is shown on the right. d, Hierarchical clustering of genes with significant changes in expression (false discovery rate $q<0.001$ ) between infections with NS1 or NS1-SUMO virus at the indicated time points (hpi). Rows show the log fold change in expression (IDs not shown; color key at bottom), and columns represent different experimental conditions. Inf, infected; un, uninfected. e, Scatter plot of cellular gene expression changes in A549 cells at 6 hpi (top) or 12 hpi (bottom) with NS1 virus or NS1-SUMO virus, relative to mockinfected (mock) cells ( $\log _{2}$ ratio). Solid and dotted lines correspond to the regression line and $95 \%$ confidence interval, respectively. Source data for a and $\mathbf{c}$ are available in Supplementary Dataset 2. 
SUMOylation of NS1 enhances suppression of the host transcriptional response via interference with RNAPII termination. Because of the known role of the CPSF complex in $3^{\prime}$-end cleavage and termination of RNAPII ${ }^{36}$, we first analyzed genome-wide RNAPII traveling ratios at transcriptional termination sites, namely the termination ratios (TRs), at both 6 and 12 hpi (Fig. 3d). This analysis indicated that downregulation of gene expression after infection is associated with a genome-wide deregulation of RNAPII $3^{\prime}$-end cleavage and termination. This deregulation was evidenced by an increase in run-through transcription, on the basis of high levels of RNA after the $3^{\prime}$ end at infection-regulated host genes at both 6 hpi (Fig. 3e) and 12 hpi (Fig. 3f). Notably, this effect was evident during NS1 virus infection and was magnified after NS1-SUMO virus infection (Fig. 3e,f). To determine whether run-through transcription might also be a feature of non-1918 viruses and dependent on NS1 expression, we used WT and NS1-deficient PR8 virus (hereafter referred to as dNS1 virus) ${ }^{6}$. Comparison between the termination ratios induced by these two viruses indicated that although WT PR8 virus infection caused RNAPII run-through transcription, this effect was abrogated in dNS1-virus-infected cells (Fig. 3g). Importantly, downregulation of Ube2L, an enzyme required for SUMOylation of substrate proteins, did not induce RNAPII termination defects (Fig. 3h). Additionally, overexpression of GFP or SUMO in uninfected cells did not induce RNAPII run-through (Fig. 3i). Runthrough was seen in infection with WT H5N1 IAV and a modified virus encoding a GFP-fused NS1 (Supplementary Fig. 4e). Finally, overexpression of SUMO in dNS1-virus-infected cells did not affect RNAPII at the termination region (Fig. 3j). Overall, these results indicate that genome-wide deregulation of RNAPII termination (i) is a feature of IAV infection, (ii) is dependent on NS1 expression, and (iii) can be controlled by SUMOylation of the 1918 NS1 SUP.

Transcriptional shut-off during infection. RNAPII run-through during infection can be functionally relevant if it is linked to repression of factors involved in the orchestration of the antiviral response or required for the viral life cycle. To explore this possibility, we analyzed the relationship between the change in run-through transcription and expression changes in NS1- versus NS1-SUMOinfected cells by cross-comparing transcript density at the $3^{\prime}$ ends of genes and the percentage of gene repression. Although virtually all genes experienced increased run-through (positive signal $>0$ for all gene curves in Supplementary Fig. 5a in NS1-SUMO versus NS1 conditions), the effect was exacerbated at genes with decreased expression in NS1-SUMO. This result suggests that run-through transcription plays a role in the downregulation of constitutively expressed genes important for cell division (for example, CDC25A and $M Y C$ ), metabolism (for example, PDK4 and RANBP6), apoptosis (for example, MCL1 and SOX4) and cell defense (for example, NFKBIA and CXCL1) (Supplementary Table 4 and representative gene-browser tracks in Supplementary Fig. 5b). Although some upregulated genes enriched in host antiviral functions (for example, IFIT1 and IFIT2) experienced run-through as well, their induction exceeded any negative effect of the increased run-through transcription seen in NS1-SUMO virus-infected cells. Notably, RNAPII run-through after infection was universally seen at all transcriptionally active genes except four (LY6E, APOL1, DEFB1, and IFI6; Supplementary Table 4), thus possibly suggesting unique features of their $3^{\prime}$ ends.

Overall, our data demonstrate that IAV infection can globally affect gene expression by deregulating RNAPII termination. This effect undermines the activity of many cell-defense genes and may be a general means by which viruses induce a state of transcriptional shut-off in infected cells.

Mechanism of run-through transcription. The defect in RNAPII cleavage and increased run-through into distal extragenic regions observed during viral infection may be the result of deregulated termination and/or other regulatory events that indirectly affect termination. To test whether this effect might be driven by inhibition of CPSF, we first reanalyzed published RNA-seq datasets in cells depleted of CPSF73, an essential subunit of the CPSF complex $^{37}$. Our analysis indicated that loss of function of CPSF activity induced RNAPII termination defects at all active genes (Fig. 4a), recapitulating the effect caused by NS1 during infection. Because CPSF also plays a key role in alternative splicing ${ }^{38-40}$, we analyzed datasets from cells treated with the splicing inhibitors spliceostatin and isoginkgetin ${ }^{41}$. Splicing inhibition induced pervasive RNAPII run-through, as evidenced by the increased run-through ratio in treated cells (Fig. 4b). These data suggest that RNAPII run-through after infection may be caused by pervasive defects in splicing rather than impaired $3^{\prime}$-end cleavage and termination. We therefore analyzed genome-wide splicing efficiencies after infection with NS1 and NS1-SUMO viruses. Global splicing defects were detected only at $12 \mathrm{hpi}$ (Fig. $4 \mathrm{c}-\mathrm{e}$ ), whereas deregulation of termination was already evident at 6 hpi (Fig. 3e). No increase in termination defects was evident in genes with multiple exons compared with monoexonic genes (Supplementary Fig. 6a). To gain further support for the idea that transcription termination defects precede splicing defects, we performed isoform sequencing (iso-seq), a method that allowed us to detect and define all splicing isoforms generated by a given gene on a genome-wide scale through long-read sequencing (details in Methods). Iso-seq of mRNA from A549 cells that were mock infected or infected with WT or NS1-SUMO virus indicated that infection did not have a pervasive effect on isoform expression at $6 \mathrm{hpi}$. This supports the idea that termination defects are the cause of transcriptional run-thorugh during infection. Despite this, infection induced some 'nonphysiological' transcripts poorly detected in mock-infected cells. These transcripts, potentially generated from interference with mRNA maturation, were increased in NS1-SUMO infection (Fig. 4f). Among the affected genes, NS1-SUMO infection had a stronger effect than NS1 infection in generating intron retention and RNAPII run-through, even for poly(A) transcripts. Furthermore, analysis of long reads mapping to viral segment 8 demonstrated that, during infection with NS1-SUMO, all mRNAs were full length (including the SUMO-encoding region) rather than truncated (Fig. 4f).

Overall, our total RNA seq and iso-seq data indicate that RNAPII run-through during IAV infection and the downregulation of most active genes is due to interference with $3^{\prime}$-end cleavage and termination, which in turn causes a defect in splicing at a later time as infection progresses. These results support the notion that defective RNAPII termination can delay RNAPII initiation and affect splicing fidelity ${ }^{42-45}$. Finally, downregulation of genes that experience RNAPII run-through in NS1-SUMO virus versus NS1 virus infected cells is likely to be caused by both delayed and decreased initiation and the direct effect of run-through RNAPII into neighboring genes, as suggested by the linear relationship between increased run-through and changes in gene expression (Supplementary Fig. 6b).

Finally, the finding that chemical inhibition of splicing causes RNAPII run-through, albeit to a lesser extent than during infection, indicates a connection between these two biological processes. This connection is further supported by evidence showing that last-exon splicing and RNAPII termination are mechanistically and evolutionarily linked events ${ }^{38,46,47}$. Overall, our data demonstrate that chemical- or virus-induced interference with cotranscriptional events can generate pervasive RNAPII run-through.

\section{Discussion}

By sampling sequences from highly pathogenic viruses, we identified the presence of a novel peptide domain comprising a SUMO site embedded in a PDZBD in the C-terminal region of NS1 expressed by the 1918 pandemic IAV strain. This SUP 
a

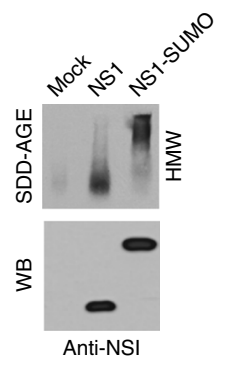

b

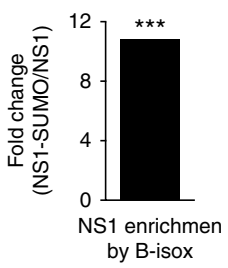

C

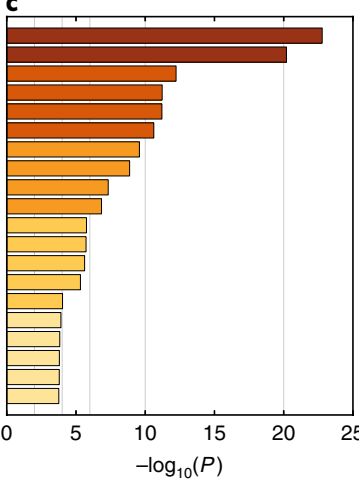

GO:0006397: mRNA processing GO:0046034: ATP metabolic process GO:0007005: mitochondrion organization GO:0034622: cellular macromoleculer complex assembly R-HSA-450408: AUF1 (hnRNP D0) binds and destabilizes mRNA GO:1902600: hydrogen ion transmembrane transport R-HSA-1268020: mitochondrial protein import (o E2 (UBE21, UBC9) GO.1002582. single-organism intracellular tranport GO:0031124: mRNA 3'-end processing aerobic respiration 0050685: positive regulation of mRNA processing O:0042407: cristae formation -HSA-380320: recruitmense to heat -HSA-72202: transport of mature tarnscript to cytoplasm GO:0006887: exocytosis GO:0000244: spliceosomal tri-snRNP complex assembly :0016236: macroautophagy

d
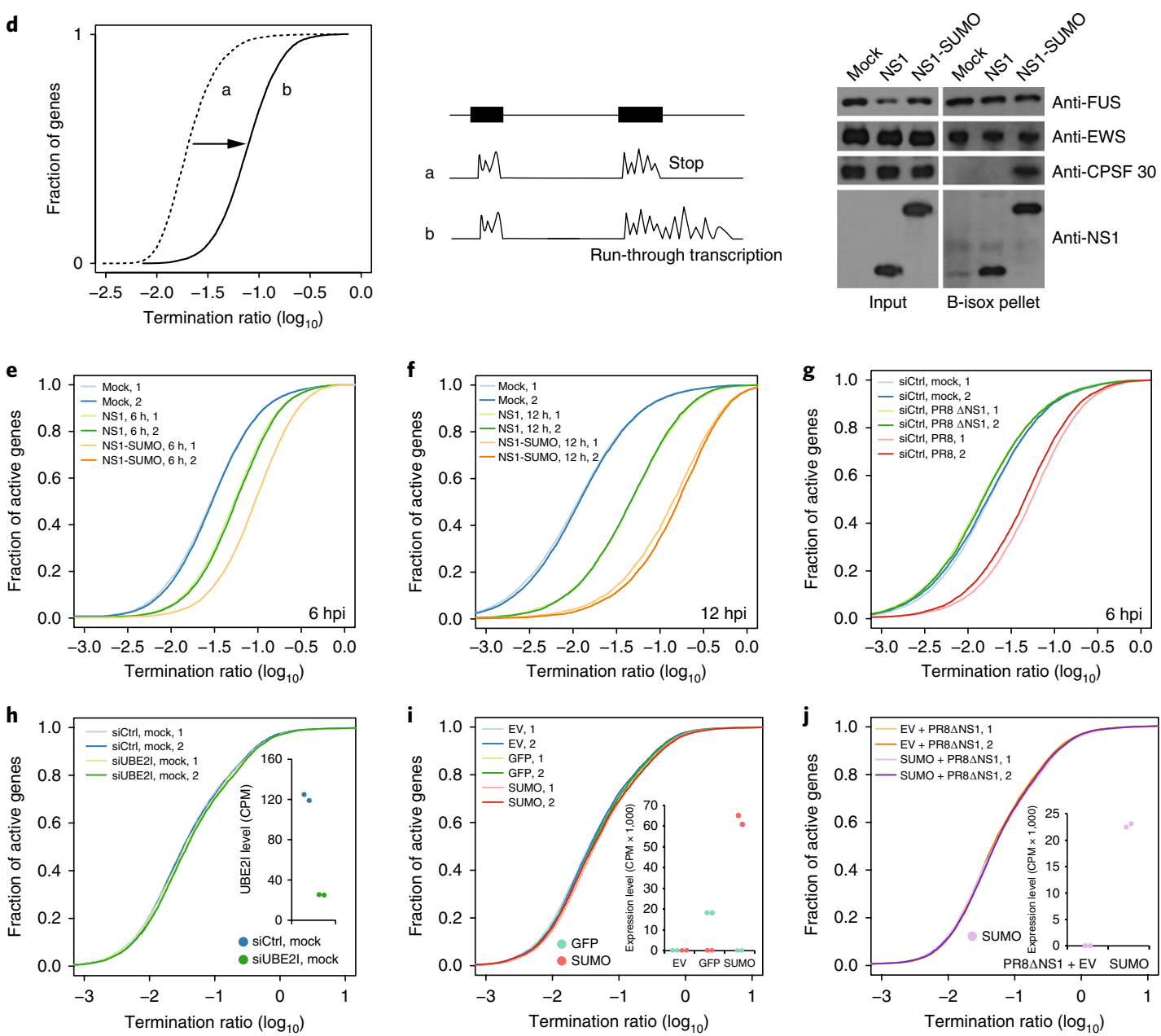

Fig. 3 | SUMOylation of NS1 induces assembly and increases pervasive RNAPII termination defects. a, SDD-AGE (top) and WB (bottom) analyses of NS1 and NS1-SUMO virus-infected lysates of A549. Mock, uninfected control. b, B-isox enrichment of NS1 from A549 lysate infected by NS1 and NS1-SUMO virus, as analyzed by MS. ${ }^{\star *} P=1.98 \times 10^{-3}$. c, Gene Ontology (GO) enrichment analysis for biological processes of B-isox-enriched proteins during NS1 and NS1-SUMO infection (top), WB of input and B-isox precipitates from NS1 and NS1-SUMO virus-infected A549 lysates with the indicated antibodies (bottom right). FUS and EWS are controls for resident RNA-granule proteins. $\mathbf{d}$, Schematic of the approach used to detect a relative increase in transcript levels in 3'-end gene-flanking regions. For each gene, a 3' transcript ratio (termination ratio, TR) was calculated as the average number of total RNA-seq reads per bp in 5-kb 3' gene-flanking regions, divided by that in exonic regions. A global relative increase in 3' transcript levels results in a horizontal transformation of the cumulative TR plot, as indicated. e,f, TR plots at termination regions of active genes (reads per kilobase per million mapped reads $($ RPKM) $>1$ in $50 \%$ of samples) in uninfected A549 cells and 6 hpi (e) or 12 hpi (f) with NS1 or NS1-SUMO virus. $\mathbf{g}$, TR plots at termination regions of active genes (RPKM $>1$ in $50 \%$ of samples) in uninfected A549 cells and at 6 hpi with PR8 or $\triangle N S 1$ virus. $\mathbf{h}-\mathbf{j}$, TR plots at the termination region of uninfected A549 cells treated with short interfering RNA against ubiquitin conjugating enzyme E2 (siUBE2I) or a control sequence (siCtrl) (h), uninfected A549 cells transfected with either empty vector (EV), or a vector expressing GFP or SUMO (i), and A549 cells infected with PR8 NNS1 and transfected with an empty vector or a vector expressing SUMO (j). The insets show the RNA expression levels of UBE2I in siCtrl- or siUBE2I- treated cells (h) and of GFP and/or SUMO in each condition (i,j) as counts per million sequenced reads (CPM). In $\mathbf{e}-\mathbf{j}$, data are shown for $n=2$ independent experiments in each condition. Uncropped blot images are shown in Supplementary Dataset 1. Source data for $\mathbf{h}-\mathbf{j}$ are available in Supplementary Dataset 2. 
a

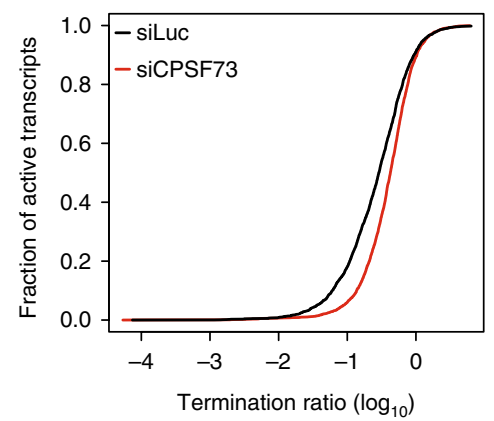

d

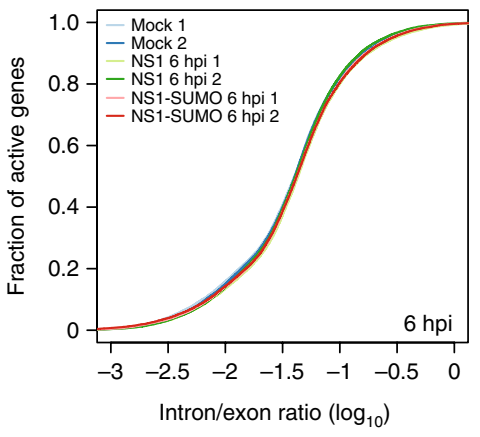

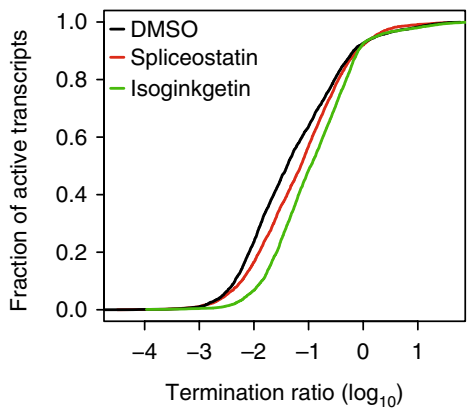

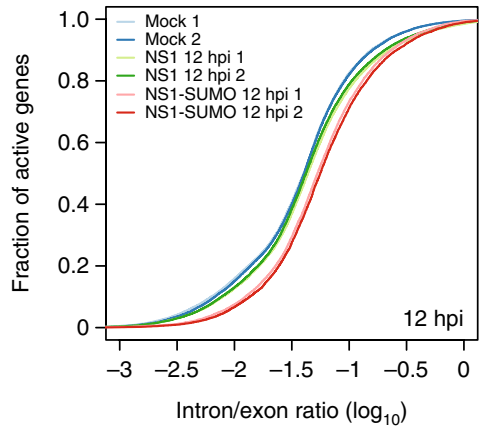

c

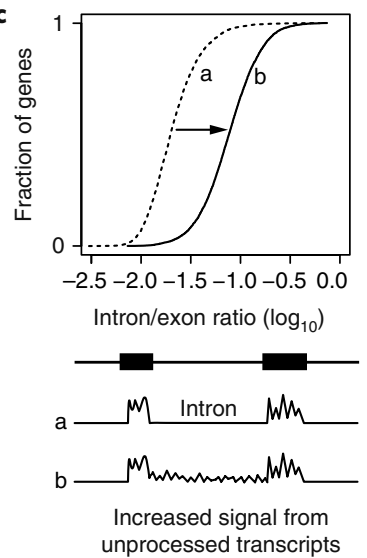

f Known/novel spliced transcripts

- Intronic/pre-mRNA transcript fragment

assible polymerase run-on fragment

Intergenic transcripts

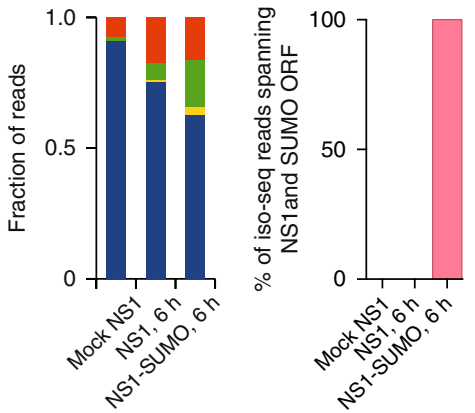

Fig. 4 | Relationship between RNAPII run-through and splicing defects. a, TR plots at the termination region in uninfected A549 cells treated with short interfering RNA against CPSF73, a subunit of the CPSF complex (siCPSF73) or a control luciferase sequence (siLuc). b, TR plots at the termination region in uninfected A549 cells treated with the indicated inhibitors and control (DMSO). c, Schematic of the approach used to detect relative accumulation of unprocessed intronic transcripts. Analogously to the TR plots, an intron/exon transcript ratio was calculated by dividing the average read coverage per bp in 5,000-bp intronic regions directly flanking an upstream exon, by the average read coverage of the upstream exon. An increase in unprocessed intronic transcripts results in horizontal transformation of the cumulative intron/exon-ratio plots, as indicated. d,e, Cumulative intron/exon ratio distributions of active genes (RPKM $>1$ in 50\% of samples) in uninfected A549 cells and 6 hpi (d) or 12 hpi (e) with NS1 or NS1-SUMO virus (e). Data are shown for two replicates in each condition. f, Classification of all iso-seq circular consensus (CCS) reads on the basis of comparisons with GENCODE v38 transcript annotations using cuffcompare (left). Percentage of reads containing full-length RNA from segment 8 in cells infected with NS1-SUMO virus (right). ORF, open reading frame. Data underlying the analysis in $\mathbf{f}$ are available in Supplementary Dataset 3.

domain confers SUMOylation of NS1. During infection, SUMO enhances NS1 association with nuclear ribonucleoprotein complexes controlling RNAPII activity. In addition, infection with the NS1-SUMO virus causes pervasive RNAPII run-through into downstream extragenic DNA and nearby genes. This result prompted us to test whether infection with other IAV strains might also cause transcription termination defects. Indeed, infection with WT PR8 virus induced RNAPII run-through, an effect also recently shown by two independent studies that mapped nascent RNA-seq ${ }^{48}$ and chromatin profiling of RNAPII ${ }^{49}$ during IAV infection with multiple strains. Unlike WT PR8, NS1-deficient virus did not induce RNAPII run-through. This result provides genetic evidence for the role of NS1 in inducing aberrant RNA biogenesis. Importantly, although NS1 proteins from different strains bind transcription regulators (such as CPSF30) with different strength in vitro, recent evidence suggests that cognate interaction between viral polymerase and NS1 can increase interaction strengths and inhibitory activity during infection ${ }^{50}$. According to this finding and our results, we speculate that all IAVs might have evolved different strategies to inhibit $3^{\prime}$-end formation. Accordingly, pathogen-derived effector proteins tend to target the same set of host factors controlling cell functions $\mathrm{s}^{51-55}$.
Effect of RNAPII run-through on gene induction and gene repression. Although most genes affected by RNAPII run-through were downregulated, some host antiviral genes were upregulated despite being affected by run-through. This result may possibly be because of unique regulatory sequences and events that control 3 ' ends. Alternatively, an extremely high induction level and burst rate at some upregulated genes might allow them to partially bypass or escape termination inhibition. Overall, our results suggest that deregulated RNAPII termination suppresses active genes and establishes transcriptional shut-off. Many viruses cause such an effect through different strategies of host interference ${ }^{56-60}$. We suggest that the different mechanisms triggering host transcriptional shut-off are linked to virus-specific requirements for their life cycle. For example, IAV requires high levels of host gene transcription (or initiation events) in the first hours after infection to mediate cap-snatching and to coordinate viral-mRNA production and viral-protein expres$\operatorname{sion}^{2}$. As such, gene suppression must be synchronized with viral replication. Overall, our results suggest that virus-induced RNAPII run-through kinetically controls host gene expression. We show that this effect is achieved by NS1 and modulated by SUMOylation in the case of the1918 strain of IAV. Constitutive NS1 SUMOylation is associated with an increased degree of RNAPII run-through at 
most active loci, concomitantly with gene suppression. Interestingly, NS1-SUMO also induces increased upregulation of some hostresponse genes. These results suggest that SUMOylation skews NS1 activity toward the control of RNAPII termination and that constitutive SUMOylation might limit other NS1 host-antagonist function. This possibility is supported by the limited infectivity of the NS1-SUMO virus in multicycle growth, as this virus cannot dynamically control this post-translational modification. In this respect, it is important to consider that SUMOylation can also be a signal for NS1 degradation. Several transcription factors are known to function transiently on chromatin and to undergo rapid turnover $^{61}$. NS1 may have adopted such a feature to provide a strategy to interfere with host transcription. Overall, our data suggest that 1918 IAV uses dynamic regulation of post-translational modifications to switch between various host-antagonizing strategies. Notably, polymorphism and mutations in SUMO-modifying enzymes exist in humans (Supplementary Fig. 6c and Supplementary Table 5), and they may potentially affect the host response to IAVs.

Evolutionary considerations. Sequence analysis of pathogenderived polypeptides can provide valuable information about sequences governing principles of protein-protein interaction ${ }^{12,62}$. Proteins encoded by viruses, especially those that have evolved to suppress the host response or co-opt cellular signaling to increase pathogen fitness, generally display multivalent interaction surfaces that allow simultaneous binding of many host proteins and nucleic acids $^{63,64}$. This functional pleiotropy can be achieved by peptide domains and IDRs ${ }^{65-68}$. Key features defining pathogen-derived linear domains and IDRs are: (i) they are located at the $\mathrm{C}$ or $\mathrm{N}$ terminus of a protein ${ }^{69}$, (ii) they are enriched in viral proteins that reside in the nuclei of infected cells $s^{62}$, and (iii) they contain post-translational-modification sites ${ }^{70}$. The SUP domain in NS1 of the 1918 IAV strain fits all these parameters. Most IDRs arise de novo via convergent evolution ${ }^{71,72}$, and the 1918 SUP is not conserved in other influenza viruses. These findings suggest that the 1918 SUP either evolved independently or was co-opted via viral mimicry. Previous work has identified how a circulating strain of IAV (H3N2) uses molecular mimicry to evolve histone-like sequences ${ }^{1}$. Although the 1918 IAV circulated for a short period of time, the NS1 SUP domain may have undergone high levels of mutagenesis and fixation in different strains. Intriguingly, the NS1-tail sequence of different strains is predictive of the host species from which the virus has been isolated ${ }^{18}$. Understanding the molecular mechanisms underlying this correlation can be informative in the context of surveillance and zoonosis $^{73}$. Notably, the only other three sequences that contain the NS1 SUP domain have been isolated from avian species, which are key host reservoirs linked to acquisition of increased pathogenicity and pandemic potential ${ }^{74,75}$. Finally, our work shows that despite being close to the centennial of its insurgence, 1918 influenza research can still instruct us about biological principles controlling pathogens and humans.

\section{Online content}

Any methods, additional references, Nature Research reporting summaries, source data, statements of data availability and associated accession codes are available at https://doi.org/10.1038/ s41594-018-0124-7.

Received: 2 November 2017; Accepted: 9 August 2018; Published online: 3 September 2018

\section{References}

1. Marazzi, I. et al. Suppression of the antiviral response by an influenza histone mimic. Nature 483, 428-433 (2012).

2. Rialdi, A. et al. The RNA exosome syncs IAV-RNAPII transcription to promote viral ribogenesis and infectivity. Cell 169, 679-692.e14 (2017).
3. Ayllon, J. \& García-Sastre, A. The NS1 protein: a multitasking virulence factor. Curr. Top. Microbiol. Immunol. 386, 73-107 (2015).

4. Gack, M. U. et al. Influenza A virus NS1 targets the ubiquitin ligase TRIM25 to evade recognition by the host viral RNA sensor RIG-I. Cell. Host. Microbe. 5, 439-449 (2009).

5. Li, S., Min, J. Y., Krug, R. M. \& Sen, G. C. Binding of the influenza A virus NS1 protein to PKR mediates the inhibition of its activation by either PACT or double-stranded RNA. Virology 349, 13-21 (2006).

6. García-Sastre, A. et al. Influenza A virus lacking the NS1 gene replicates in interferon-deficient systems. Virology 252, 324-330 (1998).

7. Donelan, N. R., Basler, C. F. \& García-Sastre, A. A recombinant influenza A virus expressing an RNA-binding-defective NS1 protein induces high levels of beta interferon and is attenuated in mice. J. Virol. 77, 13257-13266 (2003).

8. Jackson, D., Hossain, M. J., Hickman, D., Perez, D. R. \& Lamb, R. A. A new influenza virus virulence determinant: the NS1 protein four C-terminal residues modulate pathogenicity. Proc. Natl Acad. Sci. USA 105, 4381-4386 (2008).

9. Carrillo, B. et al. The influenza A virus protein NS1 displays structural polymorphism. J. Virol. 88, 4113-4122 (2014).

10. Hale, B. G. Conformational plasticity of the influenza A virus NS1 protein. J. Gen. Virol. 95, 2099-2105 (2014).

11. Davey, N. E. et al. Attributes of short linear motifs. Mol. Biosyst. 8, 268-281 (2012).

12. Gitlin, L., Hagai, T., LaBarbera, A., Solovey, M. \& Andino, R. Rapid evolution of virus sequences in intrinsically disordered protein regions. PLoS. Pathog. 10, e1004529 (2014).

13. Taubenberger, J. K. \& Morens, D. M. The pathology of influenza virus infections. Annu. Rev. Pathol. 3, 499-522 (2008).

14. Taubenberger, J. K. \& Kash, J. C. Insights on influenza pathogenesis from the grave. Virus. Res. 162, 2-7 (2011).

15. Taubenberger, J. K. et al. Reconstruction of the 1918 influenza virus: unexpected rewards from the past. mBio 3, e00201-e00212 (2012).

16. Hale, B. G., Randall, R. E., Ortín, J. \& Jackson, D. The multifunctional NS1 protein of influenza A viruses. J. Gen. Virol. 89, 2359-2376 (2008).

17. Krug, R. M. Functions of the influenza A virus NS1 protein in antiviral defense. Curr. Opin. Virol. 12, 1-6 (2015).

18. Obenauer, J. C. et al. Large-scale sequence analysis of avian influenza isolates Science 311, 1576-1580 (2006).

19. Neumann, G., Whitt, M. A. \& Kawaoka, Y. A decade after the generation of a negative-sense RNA virus from cloned cDNA: what have we learned? J. Gen. Virol. 83, 2635-2662 (2002).

20. Van Roey, K. et al. Short linear motifs: ubiquitous and functionally diverse protein interaction modules directing cell regulation. Chem. Rev. 114, 6733-6778 (2014).

21. Zhu, Q. et al. BRCA1 tumour suppression occurs via heterochromatinmediated silencing. Nature 477, 179-184 (2011).

22. Manicassamy, B. et al. Analysis of in vivo dynamics of influenza virus infection in mice using a GFP reporter virus. Proc. Natl Acad. Sci. USA 107, 11531-11536 (2010).

23. Geiss, G. K. et al. Cellular transcriptional profiling in influenza A virusinfected lung epithelial cells: the role of the nonstructural NS1 protein in the evasion of the host innate defense and its potential contribution to pandemic influenza. Proc. Natl Acad. Sci. USA 99, 10736-10741 (2002).

24. Hatada, E. \& Fukuda, R. Binding of influenza A virus NS1 protein to dsRNA in vitro. J. Gen. Virol. 73, 3325-3329 (1992).

25. Nemeroff, M. E., Qian, X. Y. \& Krug, R. M. The influenza virus NS1 protein forms multimers in vitro and in vivo. Virology 212, 422-428 (1995).

26. Bornholdt, Z. A. \& Prasad, B. V. X-ray structure of NS1 from a highly pathogenic H5N1 influenza virus. Nature 456, 985-988 (2008).

27. Molliex, A. et al. Phase separation by low complexity domains promotes stress granule assembly and drives pathological fibrillization. Cell 163, 123-133 (2015).

28. Lin, Y., Protter, D. S., Rosen, M. K. \& Parker, R. Formation and maturation of phase-separated liquid droplets by RNA-binding proteins. Mol. Cell 60 , 208-219 (2015).

29. Kwon, I. et al. Phosphorylation-regulated binding of RNA polymerase II to fibrous polymers of low-complexity domains. Cell 155, 1049-1060 (2013).

30. Banani, S. F. et al. Compositional control of phase-separated cellular bodies. Cell 166, 651-663 (2016).

31. Kato, M. et al. Cell-free formation of RNA granules: low complexity sequence domains form dynamic fibers within hydrogels. Cell 149, 753-767 (2012).

32. Harlen, K. M. \& Churchman, L. S. The code and beyond: transcription regulation by the RNA polymerase II carboxy-terminal domain. Nat. Rev. Mol. Cell Biol. 18, 263-273 (2017).

33. Heyn, P., Salmonowicz, H., Rodenfels, J. \& Neugebauer, K. M. Activation of transcription enforces the formation of distinct nuclear bodies in zebrafish embryos. RNA. Biol. 14, 752-760 (2017).

34. Hnisz, D., Shrinivas, K., Young, R. A., Chakraborty, A. K. \& Sharp, P. A. A phase separation model for transcriptional control. Cell 169, 13-23 (2017). 
35. $\mathrm{Lu}, \mathrm{H}$. et al. Phase-separation mechanism for C-terminal hyperphosphorylation of RNA polymerase II. Nature 558, 318-323 (2018).

36. Richard, P. \& Manley, J. L. Transcription termination by nuclear RNA polymerases. Genes Dev. 23, 1247-1269 (2009).

37. Schlackow, M. et al. Distinctive patterns of transcription and RNA processing for human lincRNAs. Mol. Cell 65, 25-38 (2017).

38. Martinson, H. G. An active role for splicing in $3^{\prime}$-end formation. Wiley Interdiscip. Rev. RNA 2, 459-470 (2011).

39. Misra, A., Ou, J., Zhu, L. J. \& Green, M. R. Global promotion of alternative internal exon usage by mRNA $3^{\prime}$ end formation factors. Mol. Cell 58, 819-831 (2015).

40. Misra, A. \& Green, M. R. From polyadenylation to splicing: dual role for mRNA $3^{\prime}$ end formation factors. RNA. Biol. 13, 259-264 (2016).

41. Tseng, C. K. et al. Human telomerase RNA processing and quality control. Cell Rep. 13, 2232-2243 (2015).

42. Orphanides, G. \& Reinberg, D. A unified theory of gene expression. Cell 108 439-451 (2002).

43. Perales, R. \& Bentley, D. "Cotranscriptionality": the transcription elongation complex as a nexus for nuclear transactions. Mol. Cell 36, 178-191 (2009).

44. Mapendano, C. K., Lykke-Andersen, S., Kjems, J., Bertrand, E. \& Jensen, T. H. Crosstalk between mRNA $3^{\prime}$ end processing and transcription initiation. Mol. Cell 40, 410-422 (2010).

45. Gu, B., Eick, D. \& Bensaude, O. CTD serine-2 plays a critical role in splicing and termination factor recruitment to RNA polymerase II in vivo. Nucleic Acids Res. 41, 1591-1603 (2013).

46. Niwa, M., Rose, S. D. \& Berget, S. M. In vitro polyadenylation is stimulated by the presence of an upstream intron. Genes Dev. 4, 1552-1559 (1990).

47. Dye, M. J. \& Proudfoot, N. J. Terminal exon definition occurs cotranscriptionally and promotes termination of RNA polymerase II. Mol. Cell 3, 371-378 (1999).

48. Bauer, D. L. V. et al. Influenza virus mounts a two-pronged attack on host RNA polymerase II transcription. Cell Rep. 23, 2119-2129.e3 (2018).

49. Heinz, S. et al. Transcriptional elongation can affect 3D genome architecture. Cell (in the press).

50. Kuo, R. L. \& Krug, R. M. Influenza a virus polymerase is an integral component of the CPSF30-NS1A protein complex in infected cells. J. Virol. 83, 1611-1616 (2009).

51. Pichlmair, A. et al. Viral immune modulators perturb the human molecular network by common and unique strategies. Nature 487, 486-490 (2012).

52. Rozenblatt-Rosen, $\mathrm{O}$. et al. Interpreting cancer genomes using systematic host network perturbations by tumour virus proteins. Nature 487, 491-495 (2012).

53. Marazzi, I. \& Garcia-Sastre, A. Interference of viral effector proteins with chromatin, transcription, and the epigenome. Curr. Opin. Microbiol. 26, 123-129 (2015).

54. Heaton, N. S. et al. Targeting viral proteostasis limits influenza virus, HIV, and dengue virus infection. Immunity 44, 46-58 (2016).

55. Marazzi, I., Greenbaum, B. D., Low, D. H. P. \& Guccione, E. Chromatin dependencies in cancer and inflammation. Nat. Rev. Mol. Cell Biol. 19, 245-261 (2018).

56. Maldonado, E., Cabrejos, M. E., Banks, L. \& Allende, J. E. Human papillomavirus-16 E7 protein inhibits the DNA interaction of the TATA binding transcription factor. J. Cell. Biochem. 85, 663-669 (2002).

57. Dasgupta, A. Targeting TFIIH to inhibit host cell transcription by Rift Valley Fever Virus. Mol. Cell 13, 456-458 (2004).

58. Di Valentin, E. et al. Varicella-zoster virus IE63 protein represses the basal transcription machinery by disorganizing the pre-initiation complex. Biol. Chem. 386, 255-267 (2005).

59. Kundu, P., Raychaudhuri, S., Tsai, W. \& Dasgupta, A. Shutoff of RNA polymerase II transcription by poliovirus involves $3 \mathrm{C}$ protease-mediated cleavage of the TATA-binding protein at an alternative site: incomplete shutoff of transcription interferes with efficient viral replication. J. Virol. 79, 9702-9713 (2005).

60. Fraser, K. A. \& Rice, S. A. Herpes simplex virus immediate-early protein ICP22 triggers loss of serine 2-phosphorylated RNA polymerase II. J. Virol. 81, 5091-5101 (2007).

61. Geng, F., Wenzel, S. \& Tansey, W. P. Ubiquitin and proteasomes in transcription. Annu. Rev. Biochem. 81, 177-201 (2012).

62. Hagai, T., Azia, A., Babu, M. M. \& Andino, R. Use of host-like peptide motifs in viral proteins is a prevalent strategy in host-virus interactions. Cell Rep. 7, 1729-1739 (2014).

63. Daugherty, M. D. \& Malik, H. S. Rules of engagement: molecular insights from host-virus arms races. Annu. Rev. Genet. 46, 677-700 (2012).
64. Garamszegi, S., Franzosa, E. A. \& Xia, Y. Signatures of pleiotropy, economy and convergent evolution in a domain-resolved map of human-virus protein-protein interaction networks. PLoS. Pathog. 9, e1003778 (2013).

65. Dyson, H. J. \& Wright, P. E. Intrinsically unstructured proteins and their functions. Nat. Rev. Mol. Cell Biol. 6, 197-208 (2005).

66. Uversky, V. N. \& Dunker, A. K. Understanding protein non-folding. Biochim. Biophys. Acta 1804, 1231-1264 (2010).

67. Babu, M. M., Kriwacki, R. W. \& Pappu, R. V. Versatility from protein disorder. Science 337, 1460-1461 (2012).

68. Tompa, P., Davey, N. E., Gibson, T. J. \& Babu, M. M. A million peptide motifs for the molecular biologist. Mol. Cell 55, 161-169 (2014).

69. Coletta, A. et al. Low-complexity regions within protein sequences have position-dependent roles. BMC Syst. Biol. 4, 43 (2010).

70. Beltrao, P. et al. Systematic functional prioritization of protein posttranslational modifications. Cell 150, 413-425 (2012).

71. Holt, L. J. et al. Global analysis of Cdk1 substrate phosphorylation sites provides insights into evolution. Science 325, 1682-1686 (2009).

72. Mosca, R., Pache, R. A. \& Aloy, P. The role of structural disorder in the rewiring of protein interactions through evolution. Mol. Cell. Proteomics. 11, 014969 (2012).

73. Schrauwen, E. J. \& Fouchier, R. A. Host adaptation and transmission of influenza A viruses in mammals. Emerg. Microbes Infect. 3, e9 (2014).

74. Miller, M. S. \& Palese, P. Peering into the crystal ball: influenza pandemics and vaccine efficacy. Cell 157, 294-299 (2014).

75. Taft, A. S. et al. Identification of mammalian-adapting mutations in the polymerase complex of an avian $\mathrm{H} 5 \mathrm{~N} 1$ influenza virus. Nat. Commun. 6, 7491 (2015)

\section{Acknowledgements}

We thank all members of the laboratories of I.M. and A.G.-S., and J. Bloom and A. Kornblihtt for valuable discussions and suggestions on the manuscript. We thank the Medicinal Chemistry Core, Integrated Screening Core, Microscopy CoRE,, and Global Health and Emerging Pathogens Institute (GHEPI) at the Icahn School of Medicine at Mount Sinai. H.v.B., I.M., and A.G.-S. are partially supported by HHSN272201400008CCenter for Research on Influenza Pathogenesis (CRIP), a NIAID-funded Center of Excellence for Influenza Research and Surveillance (CEIRS). I.M. is supported in part by the Department of Defense W911NF-14-1-0353. I.M. and H.v.B. are supported by NIH grant 1R01AN3663134. The findings and conclusions in this report are those of the authors and do not necessarily represent the official position of the Centers for Disease Control and Prevention/the Agency for Toxic Substances and Disease Registry. This work was supported in part through the computational resources and staff expertise provided by Scientific Computing at the Icahn School of Medicine at Mount Sinai.

\section{Author contributions}

N.Z., N. Moshkina, A.R., and V.Y. performed experiments with modeling of PDZpeptide-SUMO complexes, transfection, infection, WB, SDD-AGE, purification of recombinant proteins, BLI, pulldown assays, analysis of proteins stability, immunofluorescence, RT-PCR, chemical synthesis of B-isox, and B-isox-mediated precipitation; V.S., S.R., and B.H. performed independent validation of SUMOylation of the SUP domain; N. Mena, R.A., M.T.S.-A., J.A., and T.T. performed experiments with rescue of recombinant influenza viruses, single-cycle growth curves, plaque phenotypes, and analysis of infectious viral progeny; J.H., D.J.-M., and Y.M. performed experiments with preparation of peptides for MS, protein identification by LC-MS/MS, and statistical relative quantification of proteins and enrichment analysis; R.F., M.S., D.P., A.K., M.B., M.L.S., R.S., and H.v.B. performed directional RNA-seq, differential gene expression analysis, TR and intron/exon ratio calculation, and iso-seq analysis. S.-Y.H., D.L., and E.G. performed bioinformatics analysis on splicing and termination; B.G. performed conservation analysis; J.J., R.K.P., A.T., and N.K. provided materials and insights in experimental procedures; and A.G.-S. and I.M. supervised the project. I.M. wrote the paper, on which coauthors provided feedback.

\section{Competing interests}

The authors declare no competing interests.

\section{Additional information}

Supplementary information is available for this paper at https://doi.org/10.1038/ s41594-018-0124-7.

Reprints and permissions information is available at www.nature.com/reprints.

Correspondence and requests for materials should be addressed to I.M.

Publisher's note: Springer Nature remains neutral with regard to jurisdictional claims in published maps and institutional affiliations. 


\section{Methods}

Reagents. For chemical synthesis of B-isox, 5-(2-thienyl)-3-isoxazolecarboxylic acid (763109-71-3), $\mathrm{N}$-hydroxysuccinimide (HOSu, 6066-82-6), $\mathrm{N}$-(3-

dimethylaminopropyl)- $N^{\prime}$-ethylcarbodiimide hydrochloride (EDC- $\mathrm{HCl}, 25952$ 53-8), 6-amino-1-hexanol (4048-33-3), biotin (B4639), and 4-(dimethylamino) pyridine (DMAP, 1122-58-3) were purchased from Sigma-Aldrich. For B-isoxmediated precipitation of proteins, Halt Protease Inhibitor Cocktail (78438), Dynabeads MyOne Streptavidin C1 (65001), a SilverQuest Silver Staining Kit (LC6070), and GelCode Blue Stain Reagent (24590) were purchased from Thermo Fisher Scientific. For expression and purification of recombinant proteins, isopropyl $\beta$-D-1-thiogalactopyranoside (IPTG, I6758) and cOmplete EDTA-free Protease Inhibitor Cocktail Tablets (11873580001) were purchased from SigmaAldrich. Ni-NTA agarose (30210, Qiagen) and Nanosep Centrifugal Devices with Omega Membrane, 3K (OD003C33, Pall Corporation) were also used. For BLI, EZ-Link Sulfo-NHS-LC-biotin (21327, Thermo Fisher Scientific) and Dip and Read Streptavidin Biosensors (18-5021, ForteBio) were used. For immunofluorescence, Lipofectamine 2000 (11668027) and DAPI (D1306) were purchased from Thermo Fisher Scientific. For pulldown assays, anti-FLAG M2 Affinity Gel (A2220) was purchased from Thermo Fisher Scientific. For proteasome inhibition, MG132 (M7449) was purchased from Sigma-Aldrich. For directional RNA-seq, a RiboZero Gold rRNA Removal Kit (human/mouse/rat) (MRZG12324, Illumina), Agencourt AMPure XP (A63882, Beckman Coulter Life Sciences), TruSeq Directional Library Prep Kit (RS-122-2203, Illumina), and BluePippin 2\% M1 gels (BEF2010, Sage Scientific) were used. Antibodies were as follows. Anti-NS1 was from the laboratory of A.G.-S. Anti-FLAG (F7425, Sigma-Aldrich), anti-SUMO 2/3 (07-2167, Millipore), anti-FUS (A300-302A), anti-EWS (A300-418A), and anti-CPSF30 (A301-585A-M) were purchased from Bethyl Laboratories. Plasmids were as follows. pcDNA5 vectors encoding the indicated genes were used to express corresponding recombinant proteins in a mammalian system. pET28c vectors encoding the indicated genes were used to express recombinant proteins in a bacterial system. A549 cells (adenocarcinomic human alveolar basal epithelial cells), HEK293T cells (human embryonic kidney cells), and Madin-Darby canine kidney (MDCK) cells were originally obtained from the American Type Culture Collection. The cell cultures were tested and found to be mycoplasma-free.

Conservation analysis. All complete NS1 amino acid sequences were downloaded from the NCBI Influenza Virus Database for Human and Swine H1N1 and H3N2 and Avian H5N1. A multiple alignment was made of all sequences in the five strains and also for all sequences together. The multiple alignments were generated with multialign in MATLAB. For each group, the consensus sequence and conservation score were defined with seqconsensus in MATLAB. The average score was plotted across a ten-residue sliding window for each group of proteins and then normalized so that the maximum and minimum score were consistent across groups. These values were plotted in the accompanying heat map.

Modeling of PDZ-peptide-SUMO complexes. Initial models of the PDZ domain with NS1 peptides were generated in MODELLER 9.12 with the crystal structures of the PDZ domains or homology models when needed (Supplementary Table 1) and the sequences of the peptides ${ }^{76}$. Seven residues from the NS1 peptide were modeled (sequence RTIKSEV). The crystal structure of the Par-6 PDZ domain in complex with the Pals1 peptide was used as a template peptide for conformation in all NS1 complexes ${ }^{77}$. The initial PDZ-peptide complexes were then refined with the FlexPepDock server with default parameters ${ }^{78}$. The top-scoring model from FlexPepDock for each complex was selected, and the SUMO conjugated version was modeled by bonding the C-terminal glycine residue of the SUMO structure to the lysine residue in the peptide ${ }^{79}$. The possible spatial orientations of the conjugated SUMO domain were explored by varying the conformation of the lysine side chain in the peptides.

Transfection, infection, and western blotting. For transfected samples, $70 \%$-confluent A549 cells were transfected with the vectors encoding the indicated genes with Lipofectamine for $2 \mathrm{~d}$. The cells were washed with cold PBS twice, scraped and suspended in lysis buffer containing $20 \mathrm{mM}$ Tris- $\mathrm{HCl}, \mathrm{pH} 7.5,150 \mathrm{mM}$ $\mathrm{NaCl}, 5 \mathrm{mM} \mathrm{MgCl}_{2}, 20 \mathrm{mM} \beta$-mercaptoethanol, $0.5 \% \mathrm{NP}-40,10 \%$ glycerol, $20 \mathrm{mM}$ $\mathrm{NEM}$, and $2 \times$ Halt protease inhibitor. The cells were then lysed at $4{ }^{\circ} \mathrm{C}$ with a BIORUPTOR 300 sonicator for ten cycles of $30 \mathrm{~s}$ on and $30 \mathrm{~s}$ off at a high level of sonication. For infected samples, 100\%-confluent A549 cells were infected by the indicated viruses at the indicated MOI and collected at different time points. The cells were lysed as described above. Input and immunoprecipitated materials were analyzed by WB.

Semidenaturing detergent agarose gel electrophoresis (SDD-AGE). The infected A549 lysates were prepared as described above and centrifuged at 2,000 r.c.f. at $4{ }^{\circ} \mathrm{C}$ for $2 \mathrm{~min}$. The supernatants were removed carefully and incubated with $4 \times$ SDD-AGE loading buffer containing $2 \times$ TAE buffer, $20 \%$ glycerol, $8 \%$ SDS, and $1 \%$ bromophenol blue at room temperature for $5 \mathrm{~min}$. The samples were loaded on a $1.5 \%$ agarose gel containing $0.1 \%$ SDS. The gel was run in $1 \times$ TAE buffer with $0.1 \%$ SDS at a constant voltage of $40 \mathrm{~V}$ at $4^{\circ} \mathrm{C}$ for $3 \mathrm{~h}$. Capillary transfer and WB were then performed ${ }^{80}$.
Purification of recombinant proteins. Histidine-tagged recombinant NS1, NS1-KR, NS1-SUMO, and PSD95 proteins were overexpressed in Escherichia coli $\mathrm{BL} 21(\mathrm{DE} 3), \mathrm{OD}_{60 \mathrm{~nm}}=0.6$, with induction with IPTG at $1 \mathrm{mM}$ and incubation at $30^{\circ} \mathrm{C}$ overnight. Bacteria were harvested, washed with PBS twice, and suspended in lysis buffer containing $50 \mathrm{mM} \mathrm{NaH}_{2} \mathrm{PO}_{4}, 300 \mathrm{mM} \mathrm{NaCl}$, and $10 \mathrm{mM}$ imidazole, $\mathrm{pH}$ 8.0, with EDTA-free protease inhibitor added. The bacterial mixture was placed on ice and sonicated with a Misonix 3000 instrument for six cycles of $10 \mathrm{~s}$ on and $10 \mathrm{~s}$ off at initial output level 10 . The lysate was centrifuged at 15,000 r.p.m. at $4^{\circ} \mathrm{C}$ for $15 \mathrm{~min}$. The soluble fraction of the lysate was mixed with Ni-NTA resin and gently rotated at $4^{\circ} \mathrm{C}$ overnight. The Ni-NTA agarose was fully washed with buffer containing $50 \mathrm{mM} \mathrm{NaH}_{2} \mathrm{PO}_{4}, 300 \mathrm{mM} \mathrm{NaCl}$, and $20 \mathrm{mM}$ imidazole, pH 8.0. The proteins were finally eluted with elution buffer containing $50 \mathrm{mM} \mathrm{NaH}_{2} \mathrm{PO}_{4}$, $300 \mathrm{mM} \mathrm{NaCl}$, and $250 \mathrm{mM}$ imidazole, $\mathrm{pH}$ 8.0. The purified proteins were concentrated with Nanosep $(3 \mathrm{~K})$ centrifugal devices, and the concentrations were determined with a NanoDrop 1000 spectrophotometer. The purity of recombinant proteins was confirmed by SDS-PAGE and staining by Coomassie blue.

BLI. Recombinant PSD95 was biotinylated by reaction with Sulfo-NHS-LC-biotin with a molar coupling ratio of 1:1. Extra biotin reagent was fully removed through buffer exchange four times after the biotinylation reaction was completed. The stock of biotinylated PSD95 was diluted to $20 \mu \mathrm{g} / \mathrm{mL}$ in PBS, and stocks of NS1, NS1-KR, and NS1-SUMO were serially diluted to 10, 5, 2.5, and $1.25 \mu \mathrm{M}$ in PBS. BLI assays were performed on a ForteBio OctetRED 96 instrument. Streptavidin biosensors were washed by PBS for $60 \mathrm{~s}$, immobilized with biotinylated PSD95 $(20 \mu \mathrm{g} / \mathrm{mL})$ for $300 \mathrm{~s}$, washed a second time with PBS for $60 \mathrm{~s}$, associated with blank (PBS), NS1, NS1-KR, and NS1-SUMO at serially diluted concentrations (10, 5, 2.5 , and $1.25 \mu \mathrm{M}$ ) for $300 \mathrm{~s}$ and finally dissociated by washing with PBS for $900 \mathrm{~s}$. All steps were performed with agitation at 1,000 r.p.m. at $30^{\circ} \mathrm{C}$. BLI data were processed and analyzed in software Data Analysis 8.2. Steady-state analyses were used to generate $K_{\mathrm{d}}$ and $R^{2}$ values ${ }^{81}$.

Pulldown assays. Purified FLAG-tagged NS1, NS1-KR, and NS1-SUMO proteins were incubated with anti-FLAG agarose in PBS under rotation at $4{ }^{\circ} \mathrm{C}$ for $1 \mathrm{~h}$. The beads were fully washed with washing buffer containing $50 \mathrm{mM}$ Tris- $\mathrm{HCl}, \mathrm{pH}$ $7.5,200 \mathrm{mM} \mathrm{NaCl}, 1 \mathrm{mM}$ EDTA, and $0.2 \% \mathrm{NP}-40$ and were then washed with the same buffer without NP-40. NS1-, NS1-KR-, and NS1-SUMO-bound agarose were incubated with purified histidine-tagged PSD95 in PBS under rotation at $4^{\circ} \mathrm{C}$ for $1 \mathrm{~h}$ (and agarose washed by PBS was included as a control). The beads were fully washed with washing buffer. Bound proteins were then eluted by $3 \times$ FLAG peptide $(0.25 \mathrm{mg} / \mathrm{mL})$ by shaking the agarose at 1,000 r.p.m. at $4^{\circ} \mathrm{C}$ for $1 \mathrm{~h}$. The input and eluted proteins were run on an SDS-PAGE gel and stained by Coomassie blue.

Analysis of protein stability. A549 cells were infected with NS1 and NS1-SUMO virus for $12 \mathrm{~h}$ and were sequentially treated with $\operatorname{mg} 132(50 \mathrm{nM})$ or cycloheximide $(50 \mu \mathrm{g} / \mathrm{mL})$ for $0,4,8$, or $24 \mathrm{~h}$. Cells were lysed, and WB was performed.

Immunofluorescence. The FLAG-tagged GFP-, NS1-, NS1-KR-, and NS1-SUMOtransfected A549 lysates were washed, fixed, permeabilized, and stained with antiGFP, anti-FLAG, and DAPI. Images were observed under a Zeiss Axio Imager Z2M microscope and processed in Zen software.

Rescue of recombinant influenza viruses. Recombinant NS1 and NS1-SUMO viruses were prepared by plasmid transfection as previously described ${ }^{82}$. Two viruses rescued contained seven segments from strain A/Puerto Rico/8/34 (PR8). For the NS1-SUMO version, a modified plasmid encoding the NS segment from strain A/Brevig Mission/1/1918, in which the two viral proteins (NS1 and NEP) are encoded in separated open reading frames was used. Plasmid pDZ_NS1 encoded the original amino acid sequence, with three silent changes to allow differentiation. The plasmid pDZ_NS1-SUMO contained the SUMO2 coding sequence as a fusion at the end of the NS1 open reading frame. Rescued viruses were amplified in embryonated chicken eggs, and the NS segment was confirmed by sequencing. All work with infectious viruses containing sequences from A/ Brevig Mission/1/1918 was performed in strict accordance with CDC guidelines for biosafety level 3 (BSL3) agents at the BSL3 laboratory of the Icahn School of Medicine at Mount Sinai.

Single-cycle growth curve. Confluent monolayers of MDCK cells growing in sixwell plates were infected in triplicate with the indicated viruses at an MOI of $1 \mathrm{PFU}$ per cell. After $1 \mathrm{~h}$ adsorption, the inoculum was aspirated and replaced with $2 \mathrm{~mL}$ of infection medium (MEM supplemented with $1 \%$ antibiotics, $0.3 \%$ BSA, and $1 \mu \mathrm{g} / \mathrm{mL}$ of TPCK-treated trypsin). At the indicated times $200 \mu \mathrm{L}$ of supernatant was collected for titration and replaced with $200 \mu \mathrm{L}$ of fresh infection medium. Supernatants were titrated with hemagglutination assays with turkey red blood cells ${ }^{82}$.

Plaque phenotype. MDCK monolayers growing in six-well plates were infected with serial dilutions of the indicated virus. After $1 \mathrm{~h}$ adsorption, the inoculum was aspirated, and cells were overlaid with medium containing $0.6 \%$ agar and trypsin $(1 \mu \mathrm{g} / \mathrm{mL})$. After $2 \mathrm{~d}$, the cells were fixed with $4 \%$ formaldehyde, and plaques were visualized with a monoclonal antibody against the viral protein NP. 
Analysis of infectious viral progeny. Supernatants from a 24 -h infection were analyzed with plaque assays as described above. Isolated visible plaques were suspended in PBS and inoculated onto fresh MDCK cells. When cytopathic effects were observed, viral RNA was purified from the supernatant and amplified by RTPCR with primers specific for the NS segment. The amplified product was purified from an agarose gel and sequenced with the same primers.

RT-PCR. A549 cells were lysed with TRIzol reagent (Ambion, 15596026) and subjected to RNA extraction and DNase digestion. cDNA was synthesized with a high-capacity cDNA Reverse Transcription Kit (Applied Biosystems, 4368813) according to the manufacturer's protocol. Primers were as follows: $\beta$-actin forward, $5^{\prime}$-ACCTTCTACAATGAGCTGCG-3'; $\beta$-actin reverse, $5^{\prime}$-CCTGGATAGCAACGTACATGG-3'; GAPDH forward, $5^{\prime}$-TCTGACGCTGACTGGTTAGT; GAPDH reverse, $5^{\prime}$ -GAGGGCACAGAAAGCAATAGAG; CDC25A forward, 5' GTGGGATGGCCTTCAGATT; and CDC25A reverse, 5 CCATCAAGAACTAGGCAGAGAG.

Chemical synthesis of B-isox. B-isox was chemically synthesized according to a previously reported protocol with minor modifications ${ }^{30}$. A solution of 5-(2-thienyl)-3-isoxazolecarboxylic acid (a, $200 \mathrm{mg}, 1$ eq.), HOSu (239 mg, 2 eq.) and $\mathrm{EDC}-\mathrm{HCl}(397 \mathrm{mg}, 2$ eq.) in $5 \mathrm{~mL}$ aqueous THF was stirred at room temperature overnight to produce chemical intermediate $\mathrm{b}$. Then 6-amino1-hexanol ( $243 \mathrm{mg}, 2$ eq.) was added into the mixture, and the reaction was processing by stirring at room temperature for an additional $5 \mathrm{~h}$. Product $\mathrm{c}$ was then purified with a CombiFlash Purification System with gradient elution by $\mathrm{MeOH}$ and DCM. Chemical intermediate $\mathrm{c}(100 \mathrm{mg}, 1 \mathrm{eq}$.) was next mixed with biotin ( $85 \mathrm{mg}, 1$ eq.), DMAP (6.7 mg, 0.2 eq.), and EDC-HCl (128 mg, 2 eq.) in $5 \mathrm{~mL}$ DCM. The reaction was stirred at room temperature for $3 \mathrm{~d}$. The final product B-isox (d) was obtained with purity $\geq 98 \%$ through a CombiFlash Purification System with gradient elution with $\mathrm{MeOH}$ and DCM. The total yield was $11 \%$. B-isox was characterized with HRMS. Calculations for $\mathrm{C}_{24} \mathrm{H}_{33} \mathrm{~N}_{4} \mathrm{O}_{5} \mathrm{~S}_{2},[\mathrm{M}+\mathrm{H}]^{+}$and $\mathrm{C}_{48} \mathrm{H}_{65} \mathrm{~N}_{8} \mathrm{O}_{10} \mathrm{~S}_{4},[2 \mathrm{M}+\mathrm{H}]^{+}$were 521.1892 and $1,041.3706$, respectively, and 521.2162 and $1,041.3704$ were found.

B-isox-mediated precipitation. Six $15-\mathrm{cm}$ dishes of $100 \%$ confluent HEK293T cells were lysed as described above. The crude lysates were centrifuged at 15,000 r.p.m. at $4{ }^{\circ} \mathrm{C}$ for $10 \mathrm{~min}$ to produce six supernatants $(1 \mathrm{~mL})$. Four supernatants $(800 \mu \mathrm{L})$ were treated with B-isox $(0,10,30$, and $100 \mu \mathrm{M})$, and two supernatants were treated with B-isox $(0$ and $100 \mu \mathrm{M})$ and streptavidin-agarose as controls. The mixtures were rotated gently at $4^{\circ} \mathrm{C}$ for $1 \mathrm{~h}$. The mixtures were spun at 15,000 r.p.m. for $10 \mathrm{~min}$, and the supernatants were removed. The pellets were washed with cold lysis buffer $(20 \mathrm{mM}$ Tris- $\mathrm{HCl}, \mathrm{pH} 7.5,150 \mathrm{mM} \mathrm{NaCl}, 5 \mathrm{mM}$ $\mathrm{MgCl}_{2}, 20 \mathrm{mM} \beta$-mercaptoethanol, $0.5 \% \mathrm{NP}-40,10 \%$ glycerol, $20 \mathrm{mM} \mathrm{NEM}$, and $2 \times$ Halt protease inhibitor) twice and dissolved in $100 \mu \mathrm{L}$ of $2 \%$ SDS. The samples were boiled in $4 \times$ SDS loading buffer and run on an SDS-PAGE gel. The gel was silver stained. A549 cells were infected by mock, NS1, and NS1-SUMO viruses at an MOI of 3 for $12 \mathrm{~h}$, and the infected lysates were precipitated by $100 \mu \mathrm{M}$ B-isox as described above. The precipitated samples were analyzed by WB.

Preparation of peptides for mass spectrometry. B-isox-precipitated samples of A549 lysates infected by mock, NS1, and NS1-SUMO viruses were separated by SDS-PAGE. Gel bands for each sample were excised and then diced into small sections $\left(1 \mathrm{~mm}^{2}\right)$. Gel pieces were dehydrated in $25 \mathrm{mM} \mathrm{NH}_{4} \mathrm{HCO}_{3}$ and $50 \%$ acetonitrile $(\mathrm{ACN})$ for $10 \mathrm{~min}$ with vortexing in two consecutive rounds and were then dried under vacuum centrifugation. Slices were rehydrated in $15 \mathrm{mM}$ Tris(2carboxyethyl)phosphine (TCEP) and $25 \mathrm{mM} \mathrm{NH}_{4} \mathrm{HCO}_{3}$, and the samples were incubated for $20 \mathrm{~min}$. Freshly prepared $1 \mathrm{M}$ iodoacetamide and $25 \mathrm{mM} \mathrm{NH} \mathrm{HCO}_{3}$ were then added to attain a final concentration of $50 \mathrm{mM}$ iodoacetamide, and the samples were incubated in the dark for $20 \mathrm{~min}$. The supernatants were removed, and $25 \mathrm{mM} \mathrm{NH}_{4} \mathrm{HCO}_{3}$ was added to cover the gel pieces. The samples were vortexed for $10 \mathrm{~min}$, the supernatants were removed, and $25 \mathrm{mM} \mathrm{NH}_{4} \mathrm{HCO}_{3}$ and $50 \% \mathrm{ACN}$ were added. After incubation with vortexing for $5 \mathrm{~min}$, the supernatants were removed, and the gel slices were dried under vacuum centrifugation. A total of $0.5 \mu \mathrm{g}$ trypsin in $\mathrm{NH}_{4} \mathrm{HCO}_{3}$ was added with enough volume to cover the gel slices, and the samples were incubated at $37^{\circ} \mathrm{C}$ for digestion overnight. The supernatants containing extracted peptides were collected in separate lowbind tubes, and sufficient $50 \% \mathrm{ACN}$ and $5 \%$ formic acid (FA) were added to the remaining gel slices. Samples were vortexed for $10 \mathrm{~min}$, and the supernatants were combined with the previous extractions. This step was repeated with $100 \%$ CAN, and the supernatants were combined with the previously collected samples. Peptides were dried under vacuum centrifugation and suspended in $10 \mu \mathrm{L}$ of $3.0 \%$ ACN and $0.1 \%$ FA before MS analysis.

Protein identification by liquid chromatography with tandem mass spectrometry. Digested peptides were subjected to LC-MS/MS analysis with an Easy-nLC 1000 instrument coupled to a dual-pressure linear ion trap (Velos Pro) Orbitrap Elite mass spectrometer (Thermo Fisher Scientific). Online LC separation was performed with a fused silica IntegraFrit capillary $(75 \mathrm{~mm} \times 25 \mathrm{~cm})$ packed with $1.9 \mathrm{~mm}$ Reprosil-Pur C18 AQ reversed-phase resin. Peptides were eluted with a gradient of $5 \%$ to $30 \% \mathrm{ACN}$ in $0.1 \% \mathrm{FA}$ in $160 \mathrm{~min}$, delivered at a flow rate of $300 \mathrm{~nL} / \mathrm{minute}$. For each cycle, one full MS scan $(150-1,500 \mathrm{~m} / \mathrm{z}$, resolution of 120,000$)$ in the Orbitrap was followed by 20 data-dependent MS/MS scans fragmented by normalized collision energy (setting of 35\%) and acquired in the linear ion trap. Target ions already acquired in MS/MS scans were dynamically excluded for $20 \mathrm{~s}$. Raw MS files were analyzed by MaxQuant version 1.3.0.3 (ref. ${ }^{83}$ ), and MS/MS spectra were searched with the Andromeda search engine ${ }^{84}$ against a database containing reviewed SwissProt human and influenza protein sequences $(20,194 \text { in total })^{85}$. All runs were analyzed simultaneously to maximize the 'match between runs' algorithm available in Maxquant. The multiplicity was set to 1 (as recommended for label-free experiments), and a false discovery rate of 0.01 was imposed for peptide and protein identification.

\section{Statistical relative quantification of proteins and enrichment analysis.} Normalization of raw peptide intensities, protein-level abundance inference, and differential expression analysis ( $\log _{2}$ fold change and $P$ values) were calculated with the open source R package MSstats version 3.3.10 (ref. ${ }^{86}$ ). To ensure reproducibility, data were filtered to select for proteins identified in both biological replicas in at least one of the conditions compared. Differential expression values for proteins available in only one condition were estimated by imputation of the intensity values by random sampling from the lowest 20 normalized intensity values from the conditions in which proteins were not detected.

Gene Ontology (GO) enrichment analysis of biological processes was performed in Panther ${ }^{87}$. Data analysis and integration were carried out with the $\mathrm{R}$ language for statistical computing and graphics.

Directional RNA-seq. One microgram of DNase-treated RNA was depleted of rRNAs with a Ribo-Zero Gold rRNA Removal Kit (human/mouse/rat) according to the manufacturer's instructions and purified postdepletion with a $1.6 \times$ volume of AMPure XP beads. Barcoded directional RNA-seq libraries were then prepared with a TruSeq Directional Library Prep Kit (Illumina), according to the kit instructions. PCR products were purified with a $1.8 \times$ volume of AMPure XP beads, and fragments of 300-500 bp were size-selected with BluePippin 2\% M1 gels (Sage Scientific). Afterward, libraries were sequenced on the Illumina HiSeq 2500 platform in 100-bp single-end-read run format.

RNA-seq. After adaptor removal with cutadapt ${ }^{88}$ and base-quality trimming to remove $3^{\prime}$ read sequences if more than 20 bases with $Q<20$ were present, pairedend reads were mapped to the human (hg38) reference genome with STAR ${ }^{89}$, and gene-count summaries were generated with featureCounts ${ }^{90}$. Raw-fragment (that is, paired-end read) counts were then combined into a numeric matrix, with genes in rows and experiments in columns and used as input for differential gene expression analysis with the Bioconductor Limma package ${ }^{91}$ after multiple filtering steps to remove weakly expressed genes. First, gene counts were converted to fragments per kilobase per million reads (FPKM) with the RSEM package ${ }^{92}$ with default settings in strand-specific mode, and only genes with expression levels above 1 FPKM in at least $50 \%$ of samples were retained for further analysis. Additional filtering removed genes with fewer than 50 total reads across all samples or fewer than $200 \mathrm{nt}$ in length. Finally, normalization factors were computed on the filtered data matrix with the weighted trimmed mean of M values (TMM) method followed by voom ${ }^{93}$ mean-variance transformation in preparation for Limma linear modeling. Data were fitted to a design matrix containing all sample groups and pairwise comparisons were performed between sample groups.

Termination ratio and intron/exon-ratio calculation. To quantify an increase in transcription beyond transcription termination sites at the $3^{\prime}$ ends of genes, we calculated a measure called the TR that compares the ratio between the average read coverage in $3^{\prime}$-gene-flanking regions and the average read coverage per base pair of exonic sequence. We defined the $3^{\prime}$ flanking region between +1 and $+5,000$ bp relative to most distal $3^{\prime}$ end of annotated transcripts for known genes. Analogously, to assess the accumulation of unprocessed transcripts that might be the result of a global defect in splicing, we also calculated an intron/exon transcript density ratio for all introns. This measure was defined as the average read coverage per bp in 5,000-bp intronic regions directly flanking an upstream exon, divided by the average read coverage of the upstream exon. Transcript densities in $3^{\prime}$-gene and exon-flanking regions were calculated after exclusion of any regions overlapping annotated genes. The termination and intron/exon transcript density ratios are analogous to the TR, as described previously ${ }^{94}$.

Transcript isoform sequencing (iso-seq). RNA QC was performed with Qubit RNA HS Assay Kit (Thermo Fisher) and Agilent RNA Pico Kit protocols to assess the quantity and quality (RIN integrity) of the samples, respectively. RibosomalRNA depletion was performed according to the instructions for the Thermo Fisher Scientific RiboMinus Transcriptome Isolation Kit, human/mouse. Magnetic beads were prepared by resuspension of the RiboMinus Magnetic Beads by thorough vortexing. For each sample, $250 \mu \mathrm{l}$ of the bead suspension was pipetted into a sterile tube. The tubes were placed on a magnetic rack for $1 \mathrm{~min}$, and the supernatant was removed, discarded, and replaced with $250 \mu \mathrm{l}$ DEPC-treated 
water. The tubes were placed on a magnetic rack for $1 \mathrm{~min}$, and the supernatant was removed, discarded, and replaced with $250 \mu \mathrm{L}$ hybridization buffer. The tubes were placed on a magnetic rack for $1 \mathrm{~min}$; the supernatant was removed discarded, and replaced; and samples were resuspended in $100 \mu \mathrm{L}$ hybridization buffer. The tubes were incubated at $37^{\circ} \mathrm{C}$ until use. Hybridization was performed by combining $20 \mu \mathrm{L}$ of poly(A)-tailed total RNA, $4 \mu \mathrm{L}$ RiboMinus probe $(100 \mathrm{pmol} / \mu \mathrm{L})$, and $100 \mu \mathrm{L}$ hybridization buffer. The tubes were incubated at $37^{\circ} \mathrm{C}$ for $5 \mathrm{~min}$. The tubes were placed on ice for $30 \mathrm{~s}$ and centrifuged. $124 \mu \mathrm{L}$ of the samples was transferred to the prepared RiboMinus Magnetic beads and mixed. The tubes were incubated at $37^{\circ} \mathrm{C}$ for $15 \mathrm{~min}$. The tubes were placed on a magnetic rack for $1 \mathrm{~min}$. The supernatant was removed and transferred to a sterile tube. RNA QC was then repeated on each sample.

Isoform generation was performed according to the iso-seq templatepreparation instructions from Sequel Systems. The first-strand synthesis was performed by addition of $1-3.5 \mu \mathrm{L} 3^{\prime}$ SMART CDS primer IIA, $1-3.5 \mu \mathrm{L}$ RNA sample, and $0-2.5 \mu \mathrm{L}$ nuclease-free water, for a 4.5 total $\mu \mathrm{L}$ volume. The tubes were mixed by pipetting, centrifuged briefly, and incubated in a hot-lid thermal cycler at $72{ }^{\circ} \mathrm{C}$ for $3 \mathrm{~min}$. The temperature was slowly ramped to $42^{\circ} \mathrm{C}$ at $0.1^{\circ} \mathrm{C} / \mathrm{s}$ for $2 \mathrm{~min}$. During the incubation step, the master mix was prepared by addition of $2 \mu \mathrm{L} 5 \times$ first-strand buffer, $0.25 \mu \mathrm{L} 100 \mathrm{mM}$ DTT, $1 \mu \mathrm{L} 10 \mathrm{mM}$ dNTP, $1 \mu \mathrm{L} 12 \mu \mathrm{M}$ SMARTer II A Oligonucleotide, $0.25 \mu \mathrm{L}$ RNase inhibitor, and $1 \mu \mathrm{L}$ SMARTScribe Reverse Transcriptase, for a total volume of $5.5 \mu \mathrm{L}$ per reaction. The master mix was heated at $42^{\circ} \mathrm{C}$ for $1 \mathrm{~min}$. Aliquots of $5.5 \mu \mathrm{L}$ master mix were added each reaction tube and mixed gently by pipetting, and the tubes were spun briefly. The tubes were incubated at $42^{\circ} \mathrm{C}$ for $90 \mathrm{~min}$. The reaction was terminated by heating of the tubes at $70{ }^{\circ} \mathrm{C}$ for $10 \mathrm{~min}$. The first-strand reaction products were diluted by addition of $90 \mu \mathrm{L}$ of PacBio elution buffer.

cDNA amplification was performed as follows. $24 \times 50 \mu \mathrm{L}$ PCR reactions were set up. Per sample, PCR master mix was prepared by addition of $240 \mu \mathrm{L} 5 \times$ PrimeSTAR GXL Buffer, $240 \mu \mathrm{L}$ diluted first-strand cDNA, $96 \mu \mathrm{L}$ of $10 \mathrm{mM}$ dNTP mix for each nucleotide, $16 \mu \mathrm{L} 5^{\prime}$ PCR primer II A, $576 \mu \mathrm{L}$ nuclease-free water, and $24 \mu \mathrm{L} 1.25 \mathrm{U} / \mu \mathrm{l}$ PrimeSTAR GXL DNA polymerase. The samples were incubated at $98^{\circ} \mathrm{C}$ for $30 \mathrm{~s} ; 15$ cycles of $98^{\circ} \mathrm{C}$ for $10 \mathrm{~s}, 65^{\circ} \mathrm{C}$ for $15 \mathrm{~s}, 68^{\circ} \mathrm{C}$ for $10 \mathrm{~min}$; and a final extension at $68^{\circ} \mathrm{C}$ for $5 \mathrm{~min}$.

For PCR-product purification, the $12 \times 50 \mu \mathrm{L}$ PCR reactions were pooled, and the samples were purified with $1 \times$ AMPure PacBio (PB), fraction 1 . The remaining $12 \times 50 \mu \mathrm{L}$ PCR reactions were pooled, and the samples were purified with $0.4 \times$ AMPure PacBio, fraction 2, according to the manufacturer's protocol. The bead purifications were repeated on fractions 1 and fraction $2,1 \times$ and $0.4 \times$ volume, respectively. cDNA QC was performed with a Qubit dsDNA HS Assay Kit (Thermo Fisher), and the quality was assessed with an Agilent cDNA $12 \mathrm{~K}$ Kit, according to the manufacturers' instructions. Equal molar quantities of the two fractions per sample were pooled and subjected to SMRTbell template preparation.

SMRTbell libraries were prepared according to the manufacturer's protocol. DNA-damage repair was performed and was followed by end repair, blunt ligation of SMRTbell adaptors, and exonuclease III and IV digestion of any unligated material. SMRTbells were purified with $1 \times$ AMPure PacBio (PB) beads and eluted in $30 \mu \mathrm{L}$ of PacBio elution buffer. The samples were transferred to a fresh tube. The concentration was determined with a Qubit dsDNA HS Assay Kit (Thermo Fisher), and the quality was assessed with an Agilent cDNA 12K Kit or HS Kit, according to the manufacturers' instructions.

For sequencing, SMRTbell libraries were annealed to sequencing primer v3 sequenced with 2.1 chemistry, and 10-h movies were collected on the Sequel system according to the manufacturer's instructions. Further information on the usage of long-read sequencing can be found in ref. ${ }^{95}$ and at https://www.pacb.com/ smrt-science/smrt-sequencing/.

Statistics and reproducibility. EBayes adjusted $P$ values of RNA-seq data were corrected for multiple testing with the Benjamini-Hochberg method and used to select genes with significant expression differences. One representative western blot from of two independent experiments is shown in the main text. $t$-tests in MSstats were used to calculate $P$ values in the proteomic analysis ${ }^{86}$. Growth-curve experiments were performed two times, and the results of one representative experiment are shown in the main text.
Reporting Summary. Further information on research design is available in the Nature Research Reporting Summary linked to this article.

\section{Data availability}

RNA-seq data associated with this study are available through the Gene Expression Omnibus (GEO) data repository, accession number GSE103604. Source data for Fig. 2a,c and 3h,j are available in Supplementary Dataset 2. Data underlying the analysis in Fig. $4 \mathrm{f}$ are available in Supplementary Dataset 3. All other data are available upon reasonable request.

\section{References}

76. Eswar, N., Eramian, D., Webb, B., Shen, M. Y. \& Sali, A. Protein structure modeling with MODELLER. Methods Mol. Biol. 426, 145-159 (2008).

77. Penkert, R. R., DiVittorio, H. M. \& Prehoda, K. E. Internal recognition through PDZ domain plasticity in the Par-6-Pals1 complex. Nat. Struct. Mol. Biol. 11, 1122-1127 (2004).

78. London, N., Raveh, B., Cohen, E., Fathi, G. \& Schueler-Furman, O. Rosetta FlexPepDock web server: high resolution modeling of peptide-protein interactions. Nucleic Acids Res. 39, W249-W253 (2011).

79. Reverter, D. \& Lima, C. D. A basis for SUMO protease specificity provided by analysis of human Senp2 and a Senp2-SUMO complex. Structure 12, 1519-1531 (2004)

80. Halfmann, R. \& Lindquist, S. Screening for amyloid aggregation by semi-denaturing detergent-agarose gel electrophoresis. J. Vis. Exp. 17, e838 (2008).

81. Shah, N. B. \& Duncan, T. M. Bio-layer interferometry for measuring kinetics of protein-protein interactions and allosteric ligand effects. J. Vis. Exp. 84, e51383 (2014)

82. Martínez-Sobrido, L. \& García-Sastre, A. Generation of recombinant influenza virus from plasmid DNA. J. Vis. Exp. 42, 2057 (2010).

83. Cox, J. \& Mann, M. MaxQuant enables high peptide identification rates, individualized p.p.b.-range mass accuracies and proteome-wide protein quantification. Nat. Biotechnol. 26, 1367-1372 (2008).

84. Cox, J. et al. Andromeda: a peptide search engine integrated into the MaxQuant environment. J. Proteome. Res. 10, 1794-1805 (2011).

85. The UniProt Consortium. UniProt: the universal protein knowledgebase. Nucleic Acids Res. 45, D158-D169 (2017).

86. Choi, M. et al. MSstats: an R package for statistical analysis of quantitative mass spectrometry-based proteomic experiments. Bioinformatics 30, 2524-2526 (2014).

87. $\mathrm{Mi}, \mathrm{H}$. et al. PANTHER version 11: expanded annotation data from Gene Ontology and Reactome pathways, and data analysis tool enhancements. Nucleic Acids Res. 45, D183-D189 (2017).

88. Martin, M. Cutadapt removes adapter sequences from high-throughput sequencing reads. EMBnet. J. 17, 10-12 (2011).

89. Dobin, A. et al. STAR: ultrafast universal RNA-seq aligner. Bioinformatics 29 , 15-21 (2013).

90. Liao, Y., Smyth, G. K. \& Shi, W. featureCounts: an efficient general purpose program for assigning sequence reads to genomic features. Bioinformatics $\mathbf{3 0}$, 923-930 (2014).

91. Ritchie, M. E. et al. limma powers differential expression analyses for RNA-sequencing and microarray studies. Nucleic Acids Res. 43, e47 (2015).

92. Li, B. \& Dewey, C. N. RSEM: accurate transcript quantification from RNA-Seq data with or without a reference genome. BMC Bioinformatics 12, 323 (2011).

93. Law, C. W., Chen, Y., Shi, W. \& Smyth, G. K. voom: precision weights unlock linear model analysis tools for RNA-seq read counts. Genome. Biol. 15, R29 (2014).

94. Rahl, P. B. et al. c-Myc regulates transcriptional pause release. Cell 141, 432-445 (2010).

95. Rhoads, A. \& Au, K. F. PacBio sequencing and its applications. Genomics. Proteomics. Bioinformatics. 13, 278-289 (2015). 


\section{nature research}

\section{Reporting Summary}

Nature Research wishes to improve the reproducibility of the work that we publish. This form provides structure for consistency and transparency in reporting. For further information on Nature Research policies, see Authors \& Referees and the Editorial Policy Checklist.

\section{Statistical parameters}

When statistical analyses are reported, confirm that the following items are present in the relevant location (e.g. figure legend, table legend, main text, or Methods section).

n/a $\mid$ Confirmed

\ The exact sample size $(n)$ for each experimental group/condition, given as a discrete number and unit of measurement

$\square$ An indication of whether measurements were taken from distinct samples or whether the same sample was measured repeatedly

The statistical test(s) used AND whether they are one- or two-sided

Only common tests should be described solely by name; describe more complex techniques in the Methods section.

Х $\square$ A description of all covariates tested

$\square$ \ A description of any assumptions or corrections, such as tests of normality and adjustment for multiple comparisons

$\square$ A full description of the statistics including central tendency (e.g. means) or other basic estimates (e.g. regression coefficient) AND

variation (e.g. standard deviation) or associated estimates of uncertainty (e.g. confidence intervals)

Q For null hypothesis testing, the test statistic (e.g. $F, t, r$ ) with confidence intervals, effect sizes, degrees of freedom and $P$ value noted

$\square$ Give P values as exact values whenever suitable.

Х $\square$ For Bayesian analysis, information on the choice of priors and Markov chain Monte Carlo settings

Х $\square$ For hierarchical and complex designs, identification of the appropriate level for tests and full reporting of outcomes

Х $\square$ Estimates of effect sizes (e.g. Cohen's d, Pearson's $r$ ), indicating how they were calculated

Clearly defined error bars

State explicitly what error bars represent (e.g. SD, SE, CI)

Our web collection on statistics for biologists may be useful.

\section{Software and code}

Policy information about availability of computer code

Data collection

Data analysis

ZEN Microscopy software; Illumina HiSeq instrument and real time analysis software;

MATLAB; MODELLER v9.12; Data Analysis v8.2; FlexPepDock; MaxQuant v1.3.0.3; Andromeda search engine; MSstats v3.3.10; samtools v1.1; bcftools v1.1; R v3.3.1; fastqc 0.11.2; STAR v2.5.3a; featureCounts v1.5.0-p1; picard v2.2.4; cutadapt v1.14; limma v3.28.21; gplots v3.0.1; NMF v0.22; gProfileR v0.6.1; ggplot2 v2.2.1; GOplot v1.0.2

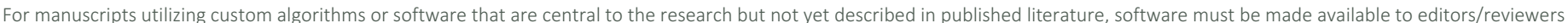

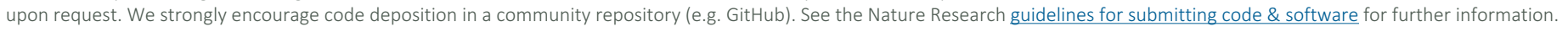

\section{Data}

Policy information about availability of data

All manuscripts must include a data availability statement. This statement should provide the following information, where applicable:

- Accession codes, unique identifiers, or web links for publicly available datasets

- A list of figures that have associated raw data

- A description of any restrictions on data availability

RNA-Seq data associated with this study is available through the Gene Expression Omnibus (GEO) data repository, accession number: GSE103604 


\section{Field-specific reporting}

Please select the best fit for your research. If you are not sure, read the appropriate sections before making your selection.

$\bigotimes$ Life sciences $\quad \square$ Behavioural \& social sciences $\quad \square$ Ecological, evolutionary \& environmental sciences

For a reference copy of the document with all sections, see nature.com/authors/policies/ReportingSummary-flat.pdf

\section{Life sciences study design}

All studies must disclose on these points even when the disclosure is negative.

Sample size All RNA-Seq experiments were performed in duplicate. The reproducibility between replicates was excellent, with $\mathrm{r} 2$ greater than 0.97 . For Iso-seq experiments we profiled only one representative sample per group.

Data exclusions No data were excluded from the study.

Replication As described in the manuscript the reproducibility of our findings was assessed using different experimental approaches and methodologies.

Randomization Where relevant, samples were allocated randomly to experimental groups.

Blinding Investigators were not blinded

\section{Behavioural \& social sciences study design}

All studies must disclose on these points even when the disclosure is negative.

Study description Briefly describe the study type including whether data are quantitative, qualitative, or mixed-methods (e.g. qualitative cross-sectional, quantitative experimental, mixed-methods case study).

Research sample

State the research sample (e.g. Harvard university undergraduates, villagers in rural India) and provide relevant demographic information (e.g. age, sex) and indicate whether the sample is representative. Provide a rationale for the study sample chosen. For studies involving existing datasets, please describe the dataset and source.

Sampling strategy Describe the sampling procedure (e.g. random, snowball, stratified, convenience). Describe the statistical methods that were used to predetermine sample size OR if no sample-size calculation was performed, describe how sample sizes were chosen and provide a rationale for why these sample sizes are sufficient. For qualitative data, please indicate whether data saturation was considered, and what criteria were used to decide that no further sampling was needed.

Data collection Provide details about the data collection procedure, including the instruments or devices used to record the data (e.g. pen and paper, computer, eye tracker, video or audio equipment) whether anyone was present besides the participant(s) and the researcher, and whether the researcher was blind to experimental condition and/or the study hypothesis during data collection.

Timing

Indicate the start and stop dates of data collection. If there is a gap between collection periods, state the dates for each sample cohort.

Data exclusions

If no data were excluded from the analyses, state so OR if data were excluded, provide the exact number of exclusions and the rationale behind them, indicating whether exclusion criteria were pre-established.

Non-participation State how many participants dropped out/declined participation and the reason(s) given OR provide response rate OR state that no participants dropped out/declined participation.

Randomization

If participants were not allocated into experimental groups, state so OR describe how participants were allocated to groups, and if allocation was not random, describe how covariates were controlled.

\section{Ecological, evolutionary \& environmental sciences study design}

All studies must disclose on these points even when the disclosure is negative.

Study description

Research sample
Briefly describe the study. For quantitative data include treatment factors and interactions, design structure (e.g. factorial, nested, hierarchical), nature and number of experimental units and replicates.

Describe the research sample (e.g. a group of tagged Passer domesticus, all Stenocereus thurberi within Organ Pipe Cactus National Monument), and provide a rationale for the sample choice. When relevant, describe the organism taxa, source, sex, age range and any manipulations. State what population the sample is meant to represent when applicable. For studies involving existing datasets, describe the data and its source. 
Data collection

Describe the data collection procedure, including who recorded the data and how.

Timing and spatial scale

Indicate the start and stop dates of data collection, noting the frequency and periodicity of sampling and providing a rationale for these choices. If there is a gap between collection periods, state the dates for each sample cohort. Specify the spatial scale from which the data are taken

Data exclusions

If no data were excluded from the analyses, state so OR if data were excluded, describe the exclusions and the rationale behind them, indicating whether exclusion criteria were pre-established.

Reproducibility

Describe the measures taken to verify the reproducibility of experimental findings. For each experiment, note whether any attempts to repeat the experiment failed OR state that all attempts to repeat the experiment were successful.

Randomization

Describe how samples/organisms/participants were allocated into groups. If allocation was not random, describe how covariates were controlled. If this is not relevant to your study, explain why

Blinding

Describe the extent of blinding used during data acquisition and analysis. If blinding was not possible, describe why OR explain why

blinding was not relevant to your study.

Did the study involve field work? $\square$ Yes $\square$ No

Field work, collection and transport

Field conditions

Describe the study conditions for field work, providing relevant parameters (e.g. temperature, rainfall).

Location

State the location of the sampling or experiment, providing relevant parameters (e.g. latitude and longitude, elevation, water depth).

Access and import/export

Describe the efforts you have made to access habitats and to collect and import/export your samples in a responsible manner and in compliance with local, national and international laws, noting any permits that were obtained (give the name of the issuing authority, the date of issue, and any identifying information).

Disturbance

Describe any disturbance caused by the study and how it was minimized.

\section{Reporting for specific materials, systems and methods}

Materials \& experimental systems

\begin{tabular}{l|l}
\hline Ia & Involved in the study \\
\hline & $\square$ Unique biological materials \\
$\square$ & Antibodies \\
$\square$ & Eukaryotic cell lines \\
$\square$ Human research participants
\end{tabular}

Methods

$\mathrm{n} / \mathrm{a}$ Involved in the study

Х ChIP-seq

$\triangle \square$ Flow cytometry

$\searrow \square$ MRI-based neuroimaging

\section{Unique biological materials}

Policy information about availability of materials

Obtaining unique materials

Describe any restrictions on the availability of unique materials $O R$ confirm that all unique materials used are readily available from the authors or from standard commercial sources (and specify these sources).

\section{Antibodies}

Antibodies used

Validation listed in methods

Abs for NS1 and SUMO were validated by overexpression of the target protein, omission of NEM or by using genetic controls (NS1 Ab was used on non infected cells).

Ab were also validated in previous independent publications by our groups and others. 
Policy information about cell lines

Cell line source(s)

ATCC

Authentication

ATCC

Mycoplasma contamination

We routinely check contamination using Mico Alert from Lonza.

RNA-seq analysis also did not identify contamination, as determined by mapping reads to representative Mycoplasma reference genomes.

Commonly misidentified lines

(See ICLAC register)

Not applicable

\section{Palaeontology}

Specimen provenance

Provide provenance information for specimens and describe permits that were obtained for the work (including the name of the issuing authority, the date of issue, and any identifying information).

Specimen deposition

Indicate where the specimens have been deposited to permit free access by other researchers.

Dating methods

If new dates are provided, describe how they were obtained (e.g. collection, storage, sample pretreatment and measurement), where they were obtained (i.e. lab name), the calibration program and the protocol for quality assurance OR state that no new dates are provided.

Tick this box to confirm that the raw and calibrated dates are available in the paper or in Supplementary Information.

\section{Animals and other organisms}

Policy information about studies involving animals; ARRIVE guidelines recommended for reporting animal research

Laboratory animals

Wild animals

Field-collected samples
For laboratory animals, report species, strain, sex and age OR state that the study did not involve laboratory animals.

Provide details on animals observed in or captured in the field; report species, sex and age where possible. Describe how animals were caught and transported and what happened to captive animals after the study (if killed, explain why and describe method; if released, say where and when) OR state that the study did not involve wild animals.

For laboratory work with field-collected samples, describe all relevant parameters such as housing, maintenance, temperature, photoperiod and end-of-experiment protocol OR state that the study did not involve samples collected from the field.

\section{Human research participants}

Policy information about studies involving human research participants

Population characteristics

Describe the covariate-relevant population characteristics of the human research participants (e.g. age, gender, genotypic information, past and current diagnosis and treatment categories). If you filled out the behavioural \& social sciences study design questions and have nothing to add here, write "See above."

Recruitment

Describe how participants were recruited. Outline any potential self-selection bias or other biases that may be present and how these are likely to impact results.

\section{ChIP-seq}

\section{Data deposition}

$\square$ Confirm that both raw and final processed data have been deposited in a public database such as $\underline{\text { GEO }}$.

$\square$ Confirm that you have deposited or provided access to graph files (e.g. BED files) for the called peaks.

Data access links

May remain private before publication.

Files in database submission

Genome browser session (e.g. $\underline{\text { UCSC }})$
For "Initial submission" or "Revised version" documents, provide reviewer access links. For your "Final submission" document, provide a link to the deposited data.

Provide a list of all files available in the database submission.

Provide a link to an anonymized genome browser session for "Initial submission" and "Revised version" documents only, to enable peer review. Write "no longer applicable" for "Final submission" documents. 
Methodology

Replicates

Describe the experimental replicates, specifying number, type and replicate agreement.

Sequencing depth

Describe the sequencing depth for each experiment, providing the total number of reads, uniquely mapped reads, length of reads and whether they were paired-or single-end.

Antibodies

Describe the antibodies used for the ChIP-seq experiments; as applicable, provide supplier name, catalog number, clone name, and lot number.

Peak calling parameters

Specify the command line program and parameters used for read mapping and peak calling, including the ChIP, control and index files used.

Data quality

Describe the methods used to ensure data quality in full detail, including how many peaks are at FDR 5\% and above 5-fold enrichment.

Software

Describe the software used to collect and analyze the ChIP-seq data. For custom code that has been deposited into a community repository, provide accession details.

\section{Flow Cytometry}

Plots

Confirm that:

$\square$ The axis labels state the marker and fluorochrome used (e.g. CD4-FITC).

The axis scales are clearly visible. Include numbers along axes only for bottom left plot of group (a 'group' is an analysis of identical markers)

All plots are contour plots with outliers or pseudocolor plots.

$\square$ A numerical value for number of cells or percentage (with statistics) is provided.

Methodology

Sample preparation

Instrument

Software

Cell population abundance

Gating strategy

Tick this box to confirm that a figure exemplifying the gating strategy is provided in the Supplementary Information.

\section{Magnetic resonance imaging}

\section{Experimental design}

\section{Design type}

Design specifications

Behavioral performance measures

Acquisition

Imaging type(s)

Field strength

Sequence \& imaging parameters

Area of acquisition

\section{Describe the sample preparation, detailing the biological source of the cells and any tissue processing steps used.} Identify the instrument used for data collection, specifying make and model number.

Describe the software used to collect and analyze the flow cytometry data. For custom code that has been deposited into a community repository, provide accession details.

Describe the abundance of the relevant cell populations within post-sort fractions, providing details on the purity of the samples and how it was determined.

Describe the gating strategy used for all relevant experiments, specifying the preliminary FSC/SSC gates of the starting cell population, indicating where boundaries between "positive" and "negative" staining cell populations are defined. 
Preprocessing

Preprocessing software

Normalization

Normalization template

Noise and artifact removal

Volume censoring

Statistical modeling \& inference

Model type and settings

Effect(s) tested

Specify type of analysis:

Statistic type for inference (See Eklund et al. 2016)

\section{Correction}

Models \& analysis

$\mathrm{n} / \mathrm{a} \mid$ Involved in the study

$\square \square$ Functional and/or effective connectivity

$\square \square$ Graph analysis

$\square \square$ Multivariate modeling or predictive analysis

Functional and/or effective connectivity

Graph analysis

Multivariate modeling and predictive analysis Carlo).
Provide detail on software version and revision number and on specific parameters (model/functions, brain extraction, segmentation, smoothing kernel size, etc.).

If data were normalized/standardized, describe the approach(es): specify linear or non-linear and define image types used for transformation $O R$ indicate that data were not normalized and explain rationale for lack of normalization.

Describe the template used for normalization/transformation, specifying subject space or group standardized space (e.g. original Talairach, MNI305, ICBM152) OR indicate that the data were not normalized.

Describe your procedure(s) for artifact and structured noise removal, specifying motion parameters, tissue signals and physiological signals (heart rate, respiration).

Define your software and/or method and criteria for volume censoring, and state the extent of such censoring.

Specify type (mass univariate, multivariate, RSA, predictive, etc.) and describe essential details of the model at the first and second levels (e.g. fixed, random or mixed effects; drift or auto-correlation).

Define precise effect in terms of the task or stimulus conditions instead of psychological concepts and indicate whether ANOVA or factorial designs were used.

Whole brain $\square$ ROI-based $\square$ Both

Specify voxel-wise or cluster-wise and report all relevant parameters for cluster-wise methods.

Describe the type of correction and how it is obtained for multiple comparisons (e.g. FWE, FDR, permutation or Monte

Report the measures of dependence used and the model details (e.g. Pearson correlation, partial correlation, mutual information).

Report the dependent variable and connectivity measure, specifying weighted graph or binarized graph, subject-or group-level, and the global and/or node summaries used (e.g. clustering coefficient, efficiency, etc.).

Specify independent variables, features extraction and dimension reduction, model, training and evaluation metrics. 